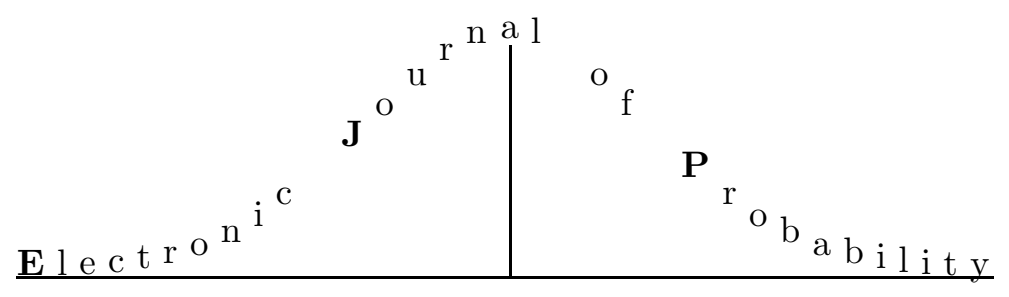

Vol. 5 (2000) Paper no. 16, pages 1-28.

Journal URL

http://www.math.washington.edu/ ejpecp/

Paper URL

http://www.math.washington.edu/ ejpecp/EjpVol5/paper16.abs.html

\title{
EIGENVALUE CURVES \\ OF ASYMMETRIC TRIDIAGONAL RANDOM MATRICES
}

Ilya Ya Goldsheid and Boris A. Khoruzhenko

School of Mathematical Sciences, Queen Mary, University of London, London E1 4NS

i.goldsheid@qmw .ac.uk, b.khoruzhenko@qmw .ac.uk

\begin{abstract}
Random Schrödinger operators with imaginary vector potentials are studied in dimension one. These operators are non-Hermitian and their spectra lie in the complex plane. We consider the eigenvalue problem on finite intervals of length $n$ with periodic boundary conditions and describe the limit eigenvalue distribution when $n \rightarrow \infty$. We prove that this limit distribution is supported by curves in the complex plane. We also obtain equations for these curves and for the corresponding eigenvalue density in terms of the Lyapunov exponent and the integrated density of states of a "reference" symmetric eigenvalue problem. In contrast to these results, the spectrum of the limit operator in $l^{2}(\mathbf{Z})$ is a two dimensional set which is not approximated by the spectra of the finite-interval operators.
\end{abstract}

Keywords random matrix, Schrödinger operator, Lyapunov exponent, eigenvalue distribution, complex eigenvalue.

AMS subject classification 82B44, 47B36, 15A52, 47B80, 47B39, 60H25, 37H15

Submitted to EJP in August 9, 2000. Final version accepted on November 21, 2000. 


\section{Introduction}

Consider an infinite asymmetric tridiagonal matrix $J$ with real entries $q_{k}$ on the main diagonal and positive entries $p_{k}$ and $r_{k}$ on the sub- and super-diagonal. Cut a square block of size $n=2 m+1$ with the center at $(0,0)$ out of $J_{n}$ and impose the periodic boundary conditions in this block. The obtained matrix has the following form

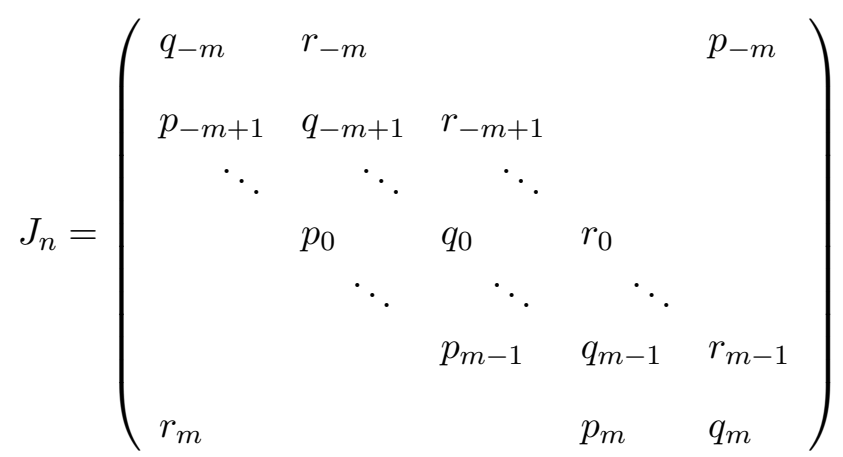

We show nonzero entries of $J_{n}$ only.

When $n$ is large, the spectrum of $J_{n}$ cannot be obtained analytically, except for some special choices of $p_{k}, q_{k}$, and $r_{k}$. However, it can be easily computed for "reasonable" values of $n$ using any of the existing linear algebra software packages. If the $p_{k}, q_{k}$, and $r_{k}$ are chosen randomly the results of such computations are striking. For large values of $n$, the spectra of $J_{n}$ lie on smooth curves which change little from sample to sample. This was observed by Hatano and Nelson $[9,10]$.
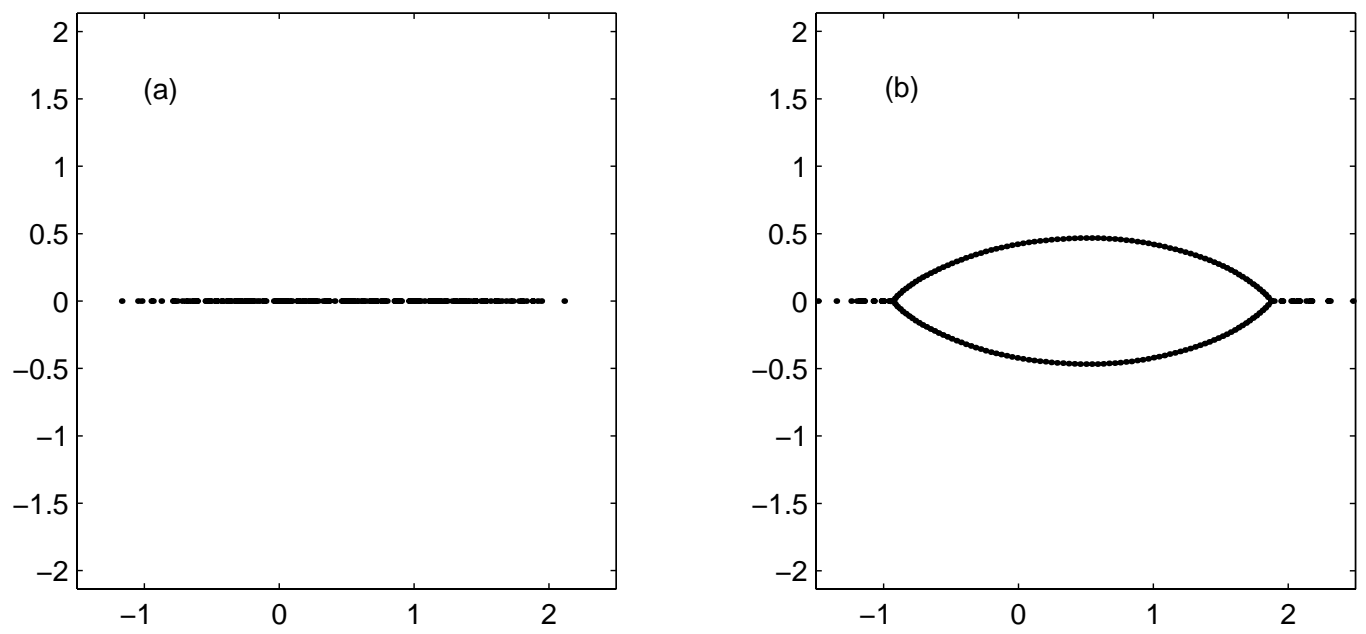

Figure 1: Spectra of $J_{n}(n=201)$ where (a) all non-zero entries are drawn from Uni[0,1]; and (b) the sub-diagonal and diagonal entries are drawn from Uni $[0,1]$ and super-diagonal entries are drawn from Uni $\left[\frac{1}{2}, 1 \frac{1}{2}\right]$.

Fig. 1 shows spectra of two matrices $J_{n}$ of dimension $n=201$. One matrix has its non-zero entries drawn from Uni $[0,1]^{1}$. Its spectrum is shown on plot (a). Note that this matrix is

\footnotetext{
${ }^{1}$ Uni $[a, b]$ denotes the uniform distribution on $[a, b]$.
} 
only stochastically symmetric. For a typical sample from Uni $[0,1], J_{n} \neq J_{n}^{T}$. Nevertheless its spectrum is real. The other matrix has its diagonal and sub-diagonal entries drawn from Uni $[0,1]$ and super-diagonal entries drawn from Uni $\left[\frac{1}{2}, 1 \frac{1}{2}\right]$. Its spectrum is shown on plot (b).

Fig. 1 is in a sharp contrast to our next figure. We took the two matrices of Fig. 1 and subtracted $\frac{1}{2}$ from all their sub- and super-diagonal entries, including the corner ones. Fig. 1 shows spectra of the obtained matrices. Note that these spectra have a two-dimensional distribution. As will soon become clear, the eigenvalue curves in Fig. 1 are due to the sub- and super-diagonal entries having the same sign.
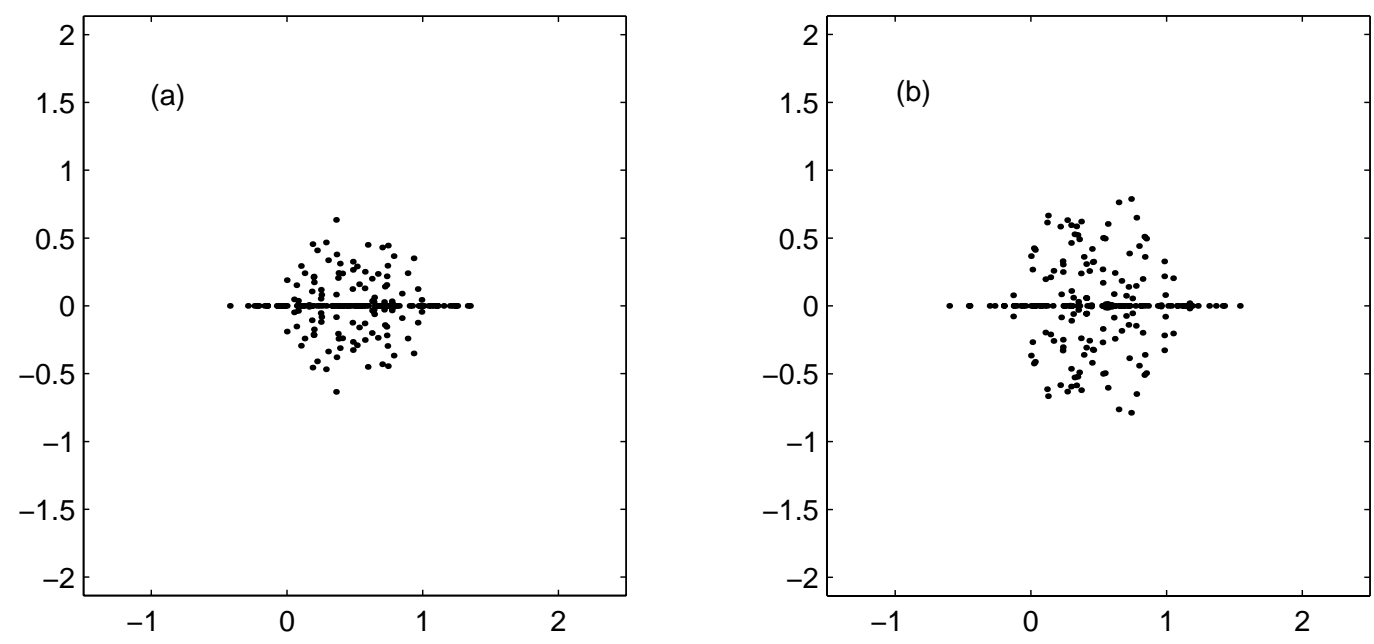

Figure 2: Spectra of $J_{n}(n=201)$ where (a) the sub- and super-diagonal entries are drawn from Uni $\left[-\frac{1}{2}, \frac{1}{2}\right]$ and the diagonal entries are drawn from Uni $[0,1]$; and (b) the sub-diagonal entries are drawn from Uni $\left[-\frac{1}{2}, \frac{1}{2}\right]$, and the diagonal and super-diagonal are drawn from Uni $[0,1]$

The class of random matrices (1.1) was introduced by Hatano and Nelson in 1996 [9, 10]. Being motivated by statistical physics of magnetic flux lines and guided by the relevant physical setup, they considered random non-Hermitian Schrödinger operators $H(g)=\left(i \frac{d}{d x}+i g\right)^{2}+V$ and their discrete analogues $J_{n}$ in a large box with periodic boundary conditions and discovered an interesting localization - delocalization transition. Hatano and Nelson also argued that the eigenvalues corresponding to the localized states are real and those corresponding to the delocalized states are non-real. Since then there has been considerable interest to the spectra of $J_{n}$ and their multi-dimensional versions in the physics literature.

Analytic descriptions of the spectrum of the random matrices $J_{n}$, in the limit $n \rightarrow \infty$, were obtained independently and almost simultaneously in $[4,3]$ and in our paper [16]. In this paper we present complete proofs of the results stated in [16]. Our results do not rely on a particular asymptotic regime (as in [4]) or a particular probability distribution (see [3]). We explain the phenomenon of eigenvalue curves depicted in Fig. 1. Under general assumptions on the probability distribution of the $p_{k}, q_{k}$, and $r_{k}$, we prove that the eigenvalues of $J_{n}$ tend to certain non-random curves in the complex plane when $n \rightarrow \infty$. We also obtain an analytic description for these curves and for the corresponding limiting distribution of eigenvalues. This limiting eigenvalue distribution may undergo a transition from a distribution on the real line, 
as in Fig. 1(a), to a distribution in the complex plane, as in Fig. 1(b), when parameter values of the probability laws of the matrix entries vary.

Our results resemble those obtained in the 1960's for truncated asymmetric Toeplitz matrices $[20,11]$. The eigenvalues of finite blocks of an infinite Toeplitz matrix are distributed along curves in the complex plane in the limit when the block size goes to infinity, see [24] for a survey. Of course, this resemblance is only formal. The class of matrices we consider is very different from Toeplitz matrices.

It is interesting that the spectra of the finite matrices $J_{n}$ with random entries, even in the limit $n \rightarrow \infty$, are entirely different from the spectrum of the corresponding infinite random matrix $J=\operatorname{tridiag}\left(p_{j}, q_{j}, r_{j}\right)$ considered as an operator acting on $l^{2}(\mathbb{Z})$. Indeed, suppose that the non-zero entries of $J$ are independently drawn from a three-dimensional distribution with a bounded support $S$. Then, the probability is one that for any given $(p, q, r) \in S \subset \mathbb{R}^{3}$ and for any $n \in \mathbb{N}$ and $\varepsilon>0$ we can always find in $J$ a block of size $n$ such that for all $j$ within this block $\left|p_{j}-p\right|<\varepsilon,\left|q_{j}-q\right|<\varepsilon$, and $\left|r_{j}-r\right|<\varepsilon$. By the Weyl criterion, this implies that with probability one the spectrum of $J=\operatorname{tridiag}\left(p_{j}, q_{j}, r_{j}\right)$ contains the $\operatorname{spectra}$ of $\operatorname{tridiag}(p, q, r)$ for all $(p, q, r) \in S$. (This argument is well known in the spectral theory of random operators, see e.g. $[5,18]$.) Since for every $(p, q, r)$ the spectrum of $\operatorname{tridiag}(p, q, r)$ is the ellipse

$$
\left\{z: z=p e^{i t}+q+r^{-i t}, t \in[0,2 \pi]\right\},
$$

the spectrum of the infinite tridiagonal random matrix $J$ has a two-dimensional support with probability one if $S$ is suffiently rich. That is why the eigenvalue curves of $J_{n}$ are surprising. This discrepancy between the spectra of $J_{n}$ and $J$ was mentioned in the preliminary account of our work [16] and was one of our motivations for studying the eigenvalue distribution for $J_{n}$. Nothing of this kind happens for periodic sequences $\left\{\left(p_{j}, q_{j}, r_{j}\right)\right\}$. If $\left\{p_{k}\right\},\left\{q_{k}\right\}$ and $\left\{r_{k}\right\}$ have a common period, then the spectrum of $J$ lies on a curve in the complex plane [17] coinciding with the eigenvalue curves of $J_{n}$ in the limit $n \rightarrow \infty$. The two-dimensional spectra are specific to "sufficiently rich" random non-selfadjoint operators (see recent works $[7,8]$ which contain a much more detailed analysis of the spectral properties of infinite tridiagonal random matrices).

One significant consequence of the above mentioned discrepancy between the spectra of $J_{n}$ and $J$ is that the norm of the resolvent $\left(J_{n}-z I_{n}\right)^{-1}$ may tend to infinity as $n \rightarrow \infty$ even when $z$ is separated from the spectra of $J_{n}$. This aspect of instability inherent in non-normal matrices $[21,23]$ was thoroughly examined for asymmetric Toeplitz matrices in [19] and for random bidiagonal matrices in [22]. We do not discuss it here.

The rest of this paper is organized as follows. Our main results, Theorems 2.1 and 2.2, are stated in Section 2. We also discuss there several corollaries of our theorems. Theorem 2.1 is proved in Section 3 and Theorem 2.2 is proved in Section 4. Our proofs use a number of results from the spectral theory of (selfadjoint) random operators. These are summarized in Appendix.

Acknowledgements We thank E.B. Davies and L.N. Trefethen for very useful and interesting discussions, and M. Sodin for bringing our attention to paper [17]. 


\section{Main results and corollaries}

To simplify the notation, we label entries of $J_{n}$ by $(j, k)$ with $j$ and $k$ being integers between 1 and $n$. We also set $p_{k}=-e^{\xi_{k-1}}$ and $r_{k}=-e^{\eta_{k}}$, so that $J_{n}$ takes the form

$$
J_{n}=\left(\begin{array}{cccc}
q_{1} & -e^{\eta_{1}} & & -e^{\xi_{0}} \\
-e^{\xi_{1}} & \ddots & \ddots & \\
& \ddots & \ddots & -e^{\eta_{n-1}} \\
-e^{\eta_{n}} & & -e^{\xi_{n-1}} & q_{n}
\end{array}\right),
$$

where only the non-zero entries of $J_{n}$ are shown ${ }^{2}$. The corresponding eigenvalue equation can be written as the second-order difference equation

$$
-e^{\xi_{k-1}} \psi_{k-1}-e^{\eta_{k}} \psi_{k+1}+q_{k} \psi_{k}=z \psi_{k}, \quad 1 \leq k \leq n,
$$

with the boundary conditions (b.c.)

$$
\psi_{0}=\psi_{n} \text { and } \psi_{n+1}=\psi_{1} .
$$

Our basic assumptions are:-

$\left\{\left(\xi_{k}, \eta_{k}, q_{k}\right)\right\}_{k=0}^{\infty}$ is a stationary ergodic (with respect to translations $k \longrightarrow k+1$ ) sequence of 3-component random vectors defined on a common probability space $(\Omega, \mathcal{F}, P)$;

$$
\mathrm{E} \ln \left(1+\left|q_{0}\right|\right), \quad \mathrm{E} \xi_{0}, \quad \mathrm{E} \eta_{0} \text { are finite. }
$$

The symbol $\mathrm{E}$ stands for the integration with respect to the probability measure, $\mathrm{E} f=\int_{\Omega} f d P$. We have chosen the off-diagonal entries of $J_{n}$ to be of the same sign. More generally, one could consider matrices whose off-diagonal entries satisfy the following condition:

$\left(^{*}\right)$ the product of $(k, k+1)$ and $(k+1, k)$ entries is positive for all $k=1,2, \ldots n-1$.

Any real asymmetric purely tridiagonal matrix satisfying $(*)$ can be transformed into a symmetric tridiagonal matrix. The recipe is well known: put $\psi_{k}=w_{k} \varphi_{k}$ in (2.2) and choose the $w_{k}$ so that to make the resulting difference equation symmetric. This is always possible when $(*)$ holds, and the weights $w_{k}$ are defined uniquely up to a multiple.

The above transformation is the starting point of our analysis. We set $w_{0}=1$ and

$$
w_{k}=e^{\frac{1}{2} \sum_{j=0}^{k-1}\left(\xi_{j}-\eta_{j}\right)}, k \geq 1 .
$$

Eqs. (2.2)-(2.3) are then transformed into

$$
\begin{gathered}
-c_{k-1} \varphi_{k-1}-c_{k} \varphi_{k+1}+q_{k} \varphi_{k}=z \varphi_{k}, \quad 1 \leq k \leq n, \\
\varphi_{n+1}=\frac{w_{1}}{w_{n+1}} \varphi_{1}, \quad \varphi_{n}=\frac{1}{w_{n}} \varphi_{0},
\end{gathered}
$$

\footnotetext{
${ }^{2}$ Following the tradition, we have chosen the sub- and super-diagonal entries to be negative. Provided the suband super-diagonal entries are of the same sign, the choice of sign does not matter.
} 
where

$$
c_{k}=e^{\frac{1}{2}\left(\xi_{k}+\eta_{k}\right)} .
$$

In the matrix form the transformation from $\psi$ to $\varphi$ can be expressed as $J_{n} \mapsto W^{-1} J_{n} W$ with $W=\operatorname{diag}\left(w_{1}, \ldots w_{n}\right)$. Eqs. (2.7) - (2.8) can be rewritten as $W^{-1} J_{n} W \varphi=z \varphi$, where $\varphi=\left(\varphi_{1}, \ldots, \varphi_{n}\right)^{T}$. The asymmetry in the transformed eigenvalue problem is due to boundary conditions (2.8). One can visualize this asymmetry: obviously,

$$
\begin{aligned}
& W^{-1} J_{n} W=H_{n}+V_{n}, \\
& H_{n}=\left(\begin{array}{cccc}
q_{1} & -c_{1} & & \\
-c_{1} & \ddots & \ddots & \\
& \ddots & \ddots & -c_{n-1} \\
& & -c_{n-1} & q_{n}
\end{array}\right) \text { and } V_{n}=\left(\begin{array}{ll} 
& a_{n} \\
& \\
b_{n} &
\end{array}\right) \text {, }
\end{aligned}
$$

where

$$
a_{n}=-c_{0} w_{n}=-c_{0} e^{\frac{1}{2} \sum_{j=0}^{n-1}\left(\xi_{j}-\eta_{j}\right)} \quad \text { and } \quad b_{n}=-c_{n} \frac{w_{1}}{w_{n+1}}=-c_{n} e^{-\frac{1}{2} \sum_{j=1}^{n}\left(\xi_{j}-\eta_{j}\right)} .
$$

$H_{n}$ is a real symmetric Jacobi matrix. Its eigenvalue equation is given by Eq. (2.7) with the Dirichlet b.c.

$$
\psi_{n+1}=\psi_{0}=0
$$

$V_{n}$, which is due to (2.8), is a real asymmetric matrix. If $\mathrm{E}\left(\xi_{0}-\eta_{0}\right) \neq 0$ then one of the two non-zero entries of $V_{n}$ increases, and the other decreases exponentially fast with $n$ (with probability 1).

Though one could deal directly with $J_{n}$, we deal with $H_{n}+V_{n}$ instead. We thus consider the asymmetric eigenvalue problem (2.7) - (2.8) as an exponentially large "perturbation" of the symmetric problem (2.7), (2.13). This point of view allows us to use the whole bulk of information about the symmetric problem (2.7), (2.13) in the context of the asymmetric problem (2.7)-(2.8). It is worth mentioning that the exponential rate of growth of $V_{n}$ is very essential: no interesting effects would be observed for sub-exponential rates.

Following the standard transfer-matrix approach, we rewrite (2.7) as

$$
\left(\begin{array}{l}
\varphi_{k+1} \\
\varphi_{k}
\end{array}\right)=A_{k}\left(\begin{array}{l}
\varphi_{k} \\
\varphi_{k-1}
\end{array}\right), \quad \text { where } A_{k}=\frac{1}{c_{k}}\left(\begin{array}{cc}
q_{k}-z & -c_{k-1} \\
c_{k} & 0
\end{array}\right)
$$

and introduce the notation

$$
S_{n}(z)=A_{n} \cdot \ldots \cdot A_{1}
$$

In order to formulate our results, we need to recall the classical notion of the Lyapunov exponent associated with Eq. (2.7):

$$
\bar{\gamma}(z)=\lim _{n \rightarrow \infty} \frac{1}{n} \mathrm{E} \log \left\|S_{n}(z)\right\|
$$

It is well known that the limit in (2.16) exists for every $z \in \mathbb{C}$ and is non-negative. Obviously any matrix norm can be used in (2.16), as they all are equivalent. It is convenient for our purposes to use the following norm

$$
\|M\|=\max _{k} \sum_{j}\left|M_{j k}\right|
$$


Introduce

$$
g=\frac{1}{2} \mathrm{E}\left(\eta_{0}-\xi_{0}\right)
$$

and consider the curve

$$
\mathcal{L}=\{z \in \mathbb{C}: \quad \bar{\gamma}(z)=|g|\} .
$$

This curve separates the two domains

$$
D_{1}=\{z \in \mathbb{C}: \quad \bar{\gamma}(z)>|g|\} \quad \text { and } \quad D_{2}=\{z \in \mathbb{C}: \quad \bar{\gamma}(z)<|g|\}
$$

in the complex plane. Note that $D_{2}$ may be empty for some values of $g$. In this case, $\mathcal{L}$ is either empty as well or degenerates into a subset of $\mathbb{R}$. In Section 3 we prove the following

Theorem 2.1 Assume (2.4) - (2.5). Then for P-almost all $\omega=\left\{\left(\xi_{j}, \eta_{j}, q_{j}\right)\right\}_{j=0}^{\infty}$ the following two statements hold:

(a) For every compact set $K_{1} \subset D_{1} \backslash \mathbb{R}$ there exists an integer number $n_{1}\left(K_{1}, \omega\right)$ such that for all $n>n_{1}\left(K_{1}, \omega\right)$ there are no eigenvalues of $J_{n}$ in $K_{1}$.

(b) For any compact set $K_{2} \subset D_{2}$ there exists an integer number $n_{2}\left(K_{2}, \omega\right)$ such that for all $n>n_{2}\left(K_{2}, \omega\right)$ there are no eigenvalues of $J_{n}$ in $K_{2}$.

To proceed, we need to introduce another well studied function, the integrated density of states $N(\lambda)$ associated with Eq. (2.7). Let

$$
N_{n}(\lambda)=\frac{1}{n} \#\left\{\text { eigenvalues of } H_{n} \text { in }(-\infty ; \lambda)\right\} .
$$

Then

$$
N(\lambda)=\lim _{n \rightarrow \infty} N_{n}(\lambda) .
$$

It is well known that under assumptions (2.4) - (2.5), the limit in Eq. (2.21) exists on a set of full probability measure and that $N(\lambda)$ is a non-random continuous function, see e.g. [5, 18].

It is a fact from spectral theory of random operators that $\bar{\gamma}(z)$ and $N(\lambda)$ are related via the Thouless formula $[5,18]$

$$
\bar{\gamma}(z)=\int_{-\infty}^{+\infty} \log |z-\lambda| d N(\lambda)-\mathrm{E} \log c_{0}
$$

According to this formula, $\bar{\gamma}(z)$, up to the additive constant $\mathrm{E} \log c_{0}=\frac{1}{2} \mathrm{E}\left(\xi_{0}+\eta_{0}\right)$, is the log-potential of $d N(\lambda)$ :

$$
\Phi(z)=\int_{-\infty}^{+\infty} \log |z-\lambda| d N(\lambda)
$$

Then $\mathcal{L}$ is an equipotential line:

$$
\mathcal{L}=\left\{z \in \mathbb{C}: \quad \Phi(z)=\max \left(\mathrm{E} \xi_{0}, \mathrm{E} \eta_{0}\right)\right\}
$$


This equipotential line consists typically of closed contours $\mathcal{L}_{j}$. In turn, each contour consists of two symmetric arcs whose endpoints lie on the real axis. The arcs are symmetric with respect to the reflection $z \mapsto \bar{z}$. The domain $D_{2}$ defined above is simply the interior of the contours $\mathcal{L}_{j}$. Part (b) of Theorem 2.1 states that for almost all $\left\{\left(\xi_{j}, \eta_{j}, q_{j}\right)\right\}_{j=0}^{\infty}$ the spectrum of $J_{n}$ is wiped out from the interior of every contour $\mathcal{L}_{j}$ as $n \rightarrow \infty$. Parts (a) and (b) together imply that for $P$-almost all $\left\{\left(\xi_{j}, \eta_{j}, q_{j}\right)\right\}_{j=0}^{\infty}$ the eigenvalues of $J_{n}$ in the limit $n \rightarrow \infty$ are located on $\mathcal{L} \cup \mathbb{R}$.

Our next result describes the limiting eigenvalue distribution on $\mathcal{L} \cup \mathbb{R}$. Let $d \nu_{J_{n}}$ denote the measure in the complex plane assigning the mass $1 / n$ to each of the $n$ eigenvalues of $J_{n}$.

Theorem 2.2 Assume (2.4) - (2.5). Then for P-almost all $\omega=\left\{\left(\xi_{j}, \eta_{j}, q_{j}\right)\right\}_{j=0}^{\infty}$ the following statement holds: For every bounded continuous function $f(z)$

$$
\lim _{n \rightarrow \infty} \int_{\mathbb{C}} f(z) d \nu_{J_{n}}(z)=\int_{\Sigma} f(\lambda) d N(\lambda)+\int_{\mathcal{L}} f(z(l)) \rho(z(l)) d l
$$

where

$$
\begin{gathered}
\Sigma=\left\{\lambda \in \mathbb{R}: \quad \lambda \in \operatorname{Supp} d N, \Phi(\lambda+i 0)>\max \left(\mathrm{E} \xi_{0}, \mathrm{E} \eta_{0}\right)\right\}, \\
\rho(z)=\frac{1}{2 \pi}\left|\int_{-\infty}^{+\infty} \frac{d N(\lambda)}{\lambda-z}\right|, \quad z \notin \mathbb{R},
\end{gathered}
$$

and $d l$ is the arc-length element on $\mathcal{L}$.

This theorem is proved in Section 4. Of course the eigenvalue curve $\mathcal{L}$ and the density of eigenvalues $\rho(z)$ on it can be found explicitly only in exceptional cases ${ }^{3}$. However, one can infer from our theorems rather detailed general information about the spectra of $J_{n}$ in the limit $n \rightarrow \infty$.

To facilitate the discussion, let us replace our basic assumption (2.4) by the following more restrictive but still quite general one:

$\left\{\left(q_{k}, \xi_{k}, \eta_{k}\right)\right\}_{k=0}^{\infty}$ is a sequence of independent identically distributed random vectors defined on a common probability space.

Under assumptions (2.4) and (2.28), the Lyapunov exponent $\bar{\gamma}(z)$ is continuous in $z$ everywhere in the complex plane, see e.g. [2]. Also, there exist positive constants $C_{0}$ and $x_{0}$ depending on the distribution of $\left(\xi_{j}, \eta_{j}, q_{j}\right)$ such that for all $|x|>x_{0}$

$$
\log |x|-C_{0}<\bar{\gamma}(x)<\log |x|+C_{0} .
$$

These inequalities are obvious if the law $F$ of distribution of $\left(\xi_{j}, \eta_{j}, q_{j}\right)$ has bounded support. If the support of $F$ is unbounded, then (2.29) can be obtained using methods of $[14,15]$. The continuity of $\bar{\gamma}(z)$ together with (2.29) imply that:

(a) $\mathcal{L}$ is not empty if and only if $\min _{x \in R} \bar{\gamma}(x) \leq|g|$;

(b) $\mathcal{L}$ is confined to a finite disk of radius $R$ depending on the distribution of $\left(\xi_{j}, \eta_{j}, q_{j}\right)$.

\footnotetext{
${ }^{3}$ One such case $[16,3]$ is when $\xi_{j} \equiv g$ and $\eta_{j} \equiv-g$ for all $j$, and the diagonal entries $q_{j}$ are Cauchy distributed.
} 
To describe $\mathcal{L}$ we notice that $\bar{\gamma}(x+i y)$ is a strictly monotone function of $y \geq 0$. This follows from the Thouless formula (2.22). Hence, if $\bar{\gamma}(x+i y)=|g|$ then

$$
\bar{\gamma}(x) \leq|g|
$$

and, vice versa, for each $x$ satisfying (2.30) one can find only one non-negative $y(x)$ such that $z=x+i y(x)$ solves the equation

$$
\bar{\gamma}(z)=|g| .
$$

Because of the continuity of $\bar{\gamma}(x)$, the set of $x$ where (2.30) holds is a union of disjoint intervals $\left[a_{j}, a_{j}^{\prime}\right]$ with $a_{j}<a_{j}^{\prime}$. Therefore $\mathcal{L}$ is a union of disconnected contours $\mathcal{L}_{j}$. Each $\mathcal{L}_{j}$ consists of two smooth arcs, $y_{j}(x)$ and $-y_{j}(x)$, formed by the solutions of Eq. (2.31) when $x$ is running over $\left[a_{j}, a_{j}^{\prime}\right]$. Apart from the specified contours, the set of solutions of Eq. (2.31) may also contain real points. These are the points where $\bar{\gamma}(x)=|g|$.

It is easy to construct examples with a prescribed finite number of contours. However we do not know any obvious reason for the number of contours to be finite for an arbitrary distribution of $\left(\xi_{j}, \eta_{j}, q_{j}\right)$.

According to (2.25), the limiting eigenvalue distribution may have two components. One, represented by the first term on the right-hand side in $(2.25)$, is supported on the real axis. We call this component real. The other, represented by the second term, is supported by $\mathcal{L}$. We call this component complex.

The following statements are simple corollaries of our Theorems. Assume (2.4) and (2.28) and consider stochastically symmetric matrices $J_{n}$, i.e. $\mathrm{E} \xi_{j}=\mathrm{E} \eta_{j}$. In this case $g=0$ and hence the curve $\mathcal{L}$ is empty. Therefore the limiting eigenvalue distribution has the real component only. This is surprising and does not seem to be obvious a priori. But even more surprising is that $\mathcal{L}$ remains empty for all

$$
|g|<g_{\mathrm{cr}}^{(1)} \equiv \min _{x \in \mathbb{R}} \bar{\gamma}(x) .
$$

If the distribution of $\left(\xi_{j}, \eta_{j}, q_{j}\right)$ is such that the support of the marginal distribution of $q_{j}$ contains at least two different points then $\bar{\gamma}(x)$ is strictly positive for all $x \in \mathbb{R}[5,18]$. In this case, by the continuity of $\bar{\gamma}(x)$ and $(2.29), g_{\mathrm{cr}}^{(1)}>0$.

On the other hand, if

$$
|g|>g_{\mathrm{cr}}^{(2)} \equiv \max _{x \in \operatorname{Supp} d N} \bar{\gamma}(x)
$$

then $\Sigma$ of (2.26) is empty and the limiting eigenvalue distribution has the complex component only. Obviously, if the law of distribution of $\left(\xi_{j}, \eta_{j}, q_{j}\right)$ has unbounded support, then $g_{\mathrm{cr}}^{(2)}=+\infty$. If $g_{\mathrm{cr}}^{(1)}<|g|<g_{\mathrm{cr}}^{(2)}$, then the real and complex components coexist.

It is worth mentioning that the density $\rho(z)$ of the non-real eigenvalues, see $(2.27)$, is analytic everywhere on $\mathcal{L}$ except the (real) end-points of the arcs. (If the limit eigenvalue distribution has no real component than $\rho(z)$ is analytic everywhere on $\mathcal{L}$.) The behavior of $\rho(z)$ near an end-point of an arc, $a_{j}$ say, depends on the regularity properties of $N(\lambda)$ at this point. If the density of states $d N(\lambda) / d \lambda$ of the reference equation (2.7) is smooth in a neighborhood of $\lambda=a_{j}$ then $\rho(z)$ has a finite limit as $z$ approaches $a_{j}$ along the arc. If, in addition, $a_{j}$ belongs to both $\Sigma$ and then the tangent to the arc at $a_{j}$ exists and is not vertical. In other words, if $d N(\lambda) / d \lambda$ is smooth in a neighborhood of a branching point $\lambda=a_{j}$ the complex branches grow out of this point linearly. 


\section{Eigenvalue curves}

In this section we prove Theorem 2.1. According to (2.10) the eigenvalues of $J_{n}$ and $H_{n}+V_{n}$ coincide. It is more convenient for us to deal with $H_{n}+V_{n}$ and we thus consider the eigenvalue problem (2.7)-(2.8). By $(2.14)-(2.15)$, we may write $\left(\varphi_{k+1}, \varphi_{k}\right)^{T}=S_{k}(z)\left(\varphi_{1}, \varphi_{0}\right)^{T}, k=$ $1, \ldots, n$, instead of $(2.7)$. In particular,

$$
\left(\begin{array}{c}
\varphi_{n+1} \\
\varphi_{n}
\end{array}\right)=S_{n}(z)\left(\begin{array}{l}
\varphi_{1} \\
\varphi_{0}
\end{array}\right)
$$

On the other hand, as required by (2.8),

$$
\left(\begin{array}{l}
\varphi_{n+1} \\
\varphi_{n}
\end{array}\right)=\frac{1}{w_{n}}\left(\begin{array}{cc}
\frac{w_{1} w_{n}}{w_{n+1}} & 0 \\
0 & 1
\end{array}\right)\left(\begin{array}{l}
\varphi_{1} \\
\varphi_{0}
\end{array}\right)
$$

Therefore the eigenvalue problem $(2.7)-(2.8)$ is equivalent to the following one:

$$
\left[\frac{1}{w_{n}} I-B_{n} S_{n}(z)\right]\left(\begin{array}{c}
\varphi_{1} \\
\varphi_{0}
\end{array}\right)=0
$$

Here $I$ is $2 \times 2$ identity matrix and

$$
B_{n}=\operatorname{diag}\left(\beta_{n}, 1\right), \quad \beta_{n}=\frac{w_{n+1}}{w_{1} w_{n}}=e^{\frac{1}{2}\left(\eta_{0}-\xi_{0}+\xi_{n}-\eta_{n}\right)} .
$$

Thus $z$ is an eigenvalue of $H_{n}+V_{n}$ if and only if

$$
\mu_{n}^{(1)}(z)=\frac{1}{w_{n}} \quad \text { or } \quad \mu_{n}^{(2)}(z)=\frac{1}{w_{n}},
$$

where $\mu_{n}^{(i)}(z), i=1,2$ are the eigenvalues of $B_{n} S_{n}(z)$.

Without loss of generality we may suppose that $g \geq 0$. Then, by the ergodic theorem,

$$
\lim _{n \rightarrow \infty} \frac{1}{n} \log \frac{1}{w_{n}} \stackrel{\text { Prob.1 }}{=} g \geq 0 .
$$

Since $\operatorname{det} S_{n}(z)=c_{0} / c_{n}$, $\operatorname{det} B_{n} S_{n}(z)$ does not depend on $z$ and, because of (3.2)and (2.9),

$$
\lim _{n \rightarrow \infty} \frac{1}{n} \log \operatorname{det} B_{n} S_{n}(z)=\lim _{n \rightarrow \infty} \frac{1}{n}\left(\eta_{0}+\eta_{n}\right) \stackrel{\text { Prob.1 }}{=} 0 .
$$

Let $r\left(B_{n} S_{n}(z)\right)$ be the spectral radius of $B_{n} S_{n}(z)$, i.e.

$$
r\left(B_{n} S_{n}(z)\right)=\max \left\{|\mu|: \quad \mu \text { is an eigenvalue of } B_{n} S_{n}(z)\right\} .
$$

From (3.3) - (3.4) we deduce a necessary condition for $z$ to be an eigenvalue of $H_{n}+V_{n}$. This condition applies to $P$-almost all $\omega \equiv\left\{\xi_{j}, \eta_{j}, q_{j}\right\}_{j=0}^{\infty}$ and is as follows: If $z$ is an eigenvalue of $H_{n}+V_{n}$ and $n>n(\omega)$ then

$$
\frac{1}{n} \log r\left(B_{n} S_{n}(z)\right)=\frac{1}{n} \log \frac{1}{w_{n}} .
$$

It is clear that this condition is necessary but not sufficient. 
We start with part (b) of Theorem 2.1. Let $K$ be an arbitrary compact subset of $D_{2}$. We shall prove that for $P$-almost all $\omega$ there is an integer $n_{0}(K, \omega)$ such that for all $n>n_{0}(K, \omega)$ equation (3.6) cannot be solved if $z \in K$.

Note that $r\left(B_{n} S_{n}(z)\right) \leq\left\|B_{n} S_{n}(z)\right\| \leq\left\|B_{n}\right\|\left\|S_{n}(z)\right\|$. Therefore,

$$
\begin{aligned}
\frac{1}{n} \log r\left(B_{n} S_{n}(z)\right) & \leq \frac{1}{n} \log \left\|B_{n}\right\|+\frac{1}{n} \log \left\|S_{n}(z)\right\| \\
& =o(1)+\frac{1}{n} \log \left\|S_{n}(z)\right\|, \quad \text { for almost all } \omega .
\end{aligned}
$$

Note that we only have to consider the case when $g>0$. For, if $g=0$ then $D_{2}=\emptyset$ and we have nothing to prove. Obviously, one can find $\varepsilon>0$ such that

$$
\sup _{z \in K} \bar{\gamma}(z) \leq g-\varepsilon .
$$

But then, by Theorem A.4 in Appendix, we have, for $P$-almost all $\omega$,

$$
\limsup _{n \rightarrow \infty}\left\{\sup _{z \in K} \frac{1}{n} \log \left\|S_{n}(z)\right\|\right\} \leq \sup _{z \in K} \bar{\gamma}(z) \leq g-\varepsilon
$$

and hence, by (3.7) - (3.8),

$$
\limsup _{n \rightarrow \infty}\left\{\sup _{z \in K} \frac{1}{n} \log r\left(B_{n} S_{n}(z)\right)\right\} \leq g-\varepsilon .
$$

Thus, for $P$-almost all $\omega$, there exists an integer $n_{1}(K, \omega)$ such that for all $n>n_{1}(K, \omega)$

$$
\frac{1}{n} \log r\left(B_{n} S_{n}(z)\right) \leq g-\frac{\varepsilon}{2} \quad \text { for all } z \in K
$$

Relations (3.9) and (3.3) contradict equation (3.6). This proves part (b) of Theorem 2.1.

Now we shall prove part (a) of Theorem 2.1. For this we need the following general (deterministic) result.

Lemma 3.1 Let $q_{1}, \ldots, q_{n}$ be real and $c_{0}, \ldots, c_{n}$ be positive. For any two complex numbers $\phi_{0}$ and $\phi_{1}$ define recursively a sequence of complex numbers $\phi_{2}, \ldots, \phi_{n+1}$ as follows:

$$
c_{k} \phi_{k+1}=\left(q_{k}-z\right) \phi_{k}-c_{k-1} \phi_{k-1}, \quad k=1,2, \ldots, n .
$$

Denote by $S_{n}$ the $2 \times 2$ matrix which maps $\left(\phi_{1}, \phi_{0}\right)^{T}$ into $\left(\phi_{n+1}, \phi_{n}\right)^{T}$ obtained as prescribed above, i.e. $S_{n}(z)=A_{n} \cdot \ldots \cdot A_{1}$, where $A_{k}$ is given by (2.14). Then for every $z \in \mathbb{C}_{+}$the matrix $S_{n}$ has two linearly independent eigenvectors $\left(u^{*}, 1\right)^{T}$ and $\left(v^{*}, 1\right)^{T}$ such that

$$
\operatorname{Im} u^{*} \leq-\frac{\operatorname{Im} z}{c_{n}}, \quad\left|u^{*}\right| \leq \frac{\left|q_{n}-z\right|}{c_{n}}+\frac{c_{n-1}^{2}}{c_{n} \operatorname{Im} z}
$$

and

$$
\operatorname{Im} v^{*} \geq 0, \quad\left|v^{*}\right| \leq \frac{c_{0}}{\operatorname{Im} z}
$$


Proof. Define $u_{1}, \ldots, u_{n+1}$ as follows: $u_{1}=u$ and $u_{k+1}=f_{k}\left(u_{k}\right), k=1, \ldots n$, where

$$
f_{k}(u)=\frac{q_{k}-z}{c_{k}}-\frac{c_{k-1}}{c_{k}} \frac{1}{u} .
$$

Obviously, $u_{n+1}=F_{n}(u)$ where $F_{n}(u)=f_{n}\left(f_{n-1}\left(\ldots f_{1}(u)\right) \ldots\right)$.

Since $\operatorname{Im} z$ and all $c_{k}$ are positive, each of the functions $f_{k}$ maps $\mathbb{C}_{-}$into itself and

$$
\operatorname{Im} f_{n}(u) \leq-\frac{\operatorname{Im} z}{c_{n}}, \quad\left|f_{n}(u)\right| \leq \frac{\left|q_{n}-z_{n}\right|}{c_{n}}+\frac{c_{n-1}}{c_{n}} \frac{1}{|u|} \quad \forall u \in \mathbb{C}_{-} .
$$

Therefore, $F_{n}$ maps continuously the compact set $Q$ defined by the inequalities (3.11) into itself. By the fixed point theorem, there exists $u^{*}$ in $Q$ such that $u^{*}=F_{n}\left(u^{*}\right)$, i.e. if $u_{n+1}=u^{*}$ given that $u_{1}=u^{*}$.

Now set $\varphi_{0}=1, \varphi_{1}=u^{*}$ and iterate (3.10) to obtain $\varphi_{k}, k=2, \ldots n+1$ using this initial data. Since $\frac{\varphi_{1}}{\varphi_{0}}=u^{*}$ and

$$
\frac{\varphi_{k+1}}{\varphi_{k}}=\frac{\left(q_{k}-z\right)}{c_{k}}-\frac{c_{k-1}}{c_{k}} \frac{\varphi_{k-1}}{\varphi_{k}}, \quad k=1,2, \ldots, n,
$$

we have that $\frac{\varphi_{k+1}}{\varphi_{k}}=u_{k+1}$ for all $k$ with the $u_{k}$ as above. In particular, $\frac{\varphi_{n+1}}{\varphi_{n}}=u_{n+1}=u^{*}$ and hence $\varphi_{n+1}=\varphi_{n} u^{*}$. But then

$$
S_{n}\left(\begin{array}{c}
u^{*} \\
1
\end{array}\right)=\varphi_{n}\left(\begin{array}{c}
u^{*} \\
1
\end{array}\right)
$$

and $\left(u^{*}, 1\right)^{T}$ is an eigenvector of $S_{n}(z)$ with $u^{*}$ satisfying (3.11).

To construct the other eigenvector one can iterate recursion (3.10) in the opposite direction. Namely, set $\varphi_{n+1}=v_{n+1}, \varphi_{n}=1$ and use (3.10) backwards to obtain the remaining $\varphi_{k}$. Similarly to what we have done before, write (3.10) as $v_{k}=\tilde{f}_{k}\left(v_{k+1}\right), k=n, \ldots 1$, where now $v_{k}=\frac{\varphi_{k}}{\varphi_{k-1}}$ and

$$
\tilde{f}_{k}(v)=\frac{c_{k-1}}{q_{k}-z-c_{k} v} .
$$

Then $v_{1}=\tilde{F}_{n}\left(v_{n+1}\right)$, where $\tilde{F}_{n}$ is the composition of $\tilde{f}_{1}, \tilde{f}_{2}, \ldots, \tilde{f}_{n}$. Each of these functions is continuous in the closure of the upper half of the complex plane and maps this set into itself. Since

$$
\left|\tilde{f}_{1}(u)\right| \leq \frac{c_{0}}{\operatorname{Im} z} \quad \forall u \in \mathbb{C}_{+},
$$

$\tilde{F}_{n}$ maps the compact set $\tilde{Q}$ defined by the inequalities in (3.12) into itself. By the fixed point theorem, there exists $v^{*} \in \tilde{Q}$ such that $\tilde{F}_{n}\left(v^{*}\right)=v^{*}$, i.e. $\varphi_{1}=v^{*} \varphi_{0}$ given that $\varphi_{n+1}=v^{*}$ and $\varphi_{n}=1$. But then

$$
S_{n}^{-1}(z)\left(\begin{array}{c}
v^{*} \\
1
\end{array}\right)=\varphi_{0}\left(\begin{array}{l}
v^{*} \\
1
\end{array}\right)
$$

and $\left(v^{*}, 1\right)^{T}$ is an eigenvector of $S_{n}(z)$ with $v^{*}$ satisfying $(3.12)$.

It is apparent that the two constructed eigenvectors are linearly independent. 
Lemma 3.2 Let $S_{n}(z)$ be as in Lemma 3.1 and $B_{n}=\operatorname{diag}\left(\beta_{n}, 1\right)$ with $\beta_{n}>0$. Then for every $z \in \mathbb{C}_{+}$the matrix $B_{n} S_{n}$ has two linearly independent eigenvectors $\left(u_{n}, 1\right)^{T}$ and $\left(v_{n}, 1\right)^{T}$ such that

$$
\operatorname{Im} u_{n} \leq-\frac{\beta_{n} \operatorname{Im} z}{c_{n}}, \quad\left|u_{n}\right| \leq \frac{\beta_{n}\left|q_{n}-z\right|}{c_{n}}+\frac{\beta_{n} c_{n-1}^{2}}{c_{n} \operatorname{Im} z}
$$

and

$$
\operatorname{Im} v_{n} \geq 0, \quad\left|v_{n}\right| \leq \frac{c_{0}}{\operatorname{Im} z}
$$

Proof. $B_{n} S_{n}(z)=\tilde{A}_{n} \cdot A_{n-1} \cdot \ldots \cdot A_{1}$, where $A_{1}, \ldots, A_{n-1}$ as before (see $\left.(2.14)-(2.15)\right)$ and

$$
\tilde{A}_{n}=\left(\begin{array}{cc}
\frac{\left(q_{n}-z\right) \beta_{n}}{c_{n}} & -\frac{c_{n-1} \beta_{n}}{c_{n}} \\
1 & 0
\end{array}\right)
$$

Since $\beta_{n}>0$, Lemma 3.1 applies.

We now return to our eigenvalue problem $(2.7)-(2.8)$ and to the matrices $S_{n}(z)$ and $B_{n}$ associated with this problem, i.e. now $c_{k}$ are given by (2.9) and $\beta_{n}$ by (3.2). Set

$$
T_{n}=\left(\begin{array}{ll}
u_{n} & v_{n} \\
1 & 1
\end{array}\right)
$$

where $\left(u_{n}, 1\right)^{T}$ and $\left(v_{n}, 1\right)^{T}$ are the eigenvectors of $B_{n} S_{n}(z)$ obtained in Lemma 3.2.

Lemma 3.3 Assume (2.4) - (2.5). Then for P-almost all $\left\{\xi_{k}, \eta_{k}, q_{k}\right\}_{k=0}^{\infty}$ the following statement holds: For all $z \in \mathbb{C}_{+}$

$$
\lim _{n \rightarrow \infty} \frac{1}{n} \log \left\|T_{n}\right\|\left\|T_{n}^{-1}\right\|=0 .
$$

The convergence in (3.18) is uniform in $z$ on every compact set in $\mathbb{C}_{+}$.

Proof. For any stationary ergodic sequence of random variables $X_{n}$ with finite first moment $\lim _{n \rightarrow \infty} X_{n} / n=0$ with probability 1 . By $(2.17)$,

$$
0 \leq \frac{1}{n} \log \left\|T_{n}|||| T_{n}^{-1}\right\| \leq \frac{1}{n} \log \frac{\left(\left|u_{n}\right|+\left|v_{n}\right|+2\right)^{2}}{\left|u_{n}-v_{n}\right|} .
$$

To complete the proof, apply inequalities (3.15) - (3.16).

Recall that $r\left(B_{n} S_{n}(z)\right)$ is used to denote the spectral radius (3.5).

Lemma 3.4 Assume (2.4) - (2.5). Then for P-almost all $\left\{\xi_{k}, \eta_{k}, q_{k}\right\}_{k=0}^{\infty}$ the following statement holds: For all $z \in \mathbb{C}_{+}$

$$
\lim _{n \rightarrow \infty} \frac{1}{n} \log r\left(B_{n} S_{n}(z)\right)=\bar{\gamma}(z),
$$

where $\bar{\gamma}(z)$ is the Lyapunov exponent (2.16). The convergence in (3.18) is uniform on every compact set in $\mathbb{C}_{+}$. 
Proof. It follows from Lemma 3.2 that $B_{n} S_{n}(z)=T_{n} \Lambda_{n} T_{n}^{-1}$, where $\Lambda_{n}$ is the diagonal matrix of eigenvalues of $B_{n} S_{n}(z)$ corresponding to $T_{n}$. Then

$$
\frac{1}{\left\|T_{n}\right\|\left\|T_{n}^{-1}\right\|} \leq \frac{\left\|B_{n} S_{n}\right\|}{\left\|\Lambda_{n}\right\|} \leq\left\|T_{n}\right\|\left\|T_{n}^{-1}\right\|
$$

With our choice (2.17) of the matrix norm, $\|\Lambda\|=r\left(B_{n} S_{n}(z)\right)$ and, by Lemma 3.3, for $P$-almost all $\left\{\xi_{k}, \eta_{k}, q_{k}\right\}_{k=0}^{\infty}$,

$$
\lim _{n \rightarrow \infty} \frac{1}{n} \log \frac{\left\|B_{n} S_{n}(z)\right\|}{r\left(B_{n} S_{n}(z)\right)}=0 \quad \text { uniformly in } z \text { on compact subsets of } \mathbb{C}_{+} .
$$

On the other hand, for $P$-almost all $\left\{\xi_{k}, \eta_{k}, q_{k}\right\}_{k=0}^{\infty}$,

$$
\lim _{n \rightarrow \infty} \frac{1}{n} \log \frac{\left\|B_{n} S_{n}(z)\right\|}{\left\|S_{n}(z)\right\|}=0 \quad \text { uniformly in } z
$$

This follows from the obvious inequalities

$$
\frac{\left\|S_{n}(z)\right\|}{\left\|B_{n}^{-1}\right\|} \leq\left\|B_{n} S_{n}(z)\right\| \leq\left\|B_{n}\right\|\left\|S_{n}(z)\right\| .
$$

Now the statement of Lemma follows from Theorem A.3 of Appendix.

With Lemma 3.4 in hand, we are a in a position to prove part (a) of Theorem 2.1. Let $K$ be a compact subset of $D_{1} \backslash \mathbb{R}$. As $\bar{\gamma}(z)$ is continuous in $K$ and $\bar{\gamma}(z)>g$ there, one can find an $\varepsilon>0$ such that

$$
\min _{z \in K} \bar{\gamma}(z) \geq g+\varepsilon \text {. }
$$

From this, by Lemma 3.4, for almost all $\omega=\left\{\xi_{k}, \eta_{k}, q_{k}\right\}_{k=0}^{\infty}$, there exists an integer $n_{1}(K, \omega)$ such that for all $n>n_{1}(K, \omega)$

$$
\frac{1}{n} \log r\left(B_{n} S_{n}(z)\right) \geq g+\frac{\varepsilon}{2} \quad \forall z \in K
$$

In view of $(3.3),(3.20)$ contradicts (3.6). Theorem 2.1 is proved.

\section{Distribution of eigenvalues}

We need to introduce more notations and to recall few elementary facts from potential theory. Let $M_{n}$ be an $n \times n$ matrix. We denote by $d \nu_{M_{n}}$ the measure on $\mathbb{C}$ that assigns to each of the $n$ eigenvalues of $M_{n}$ the mass $\frac{1}{n}$. This measure describes the distribution of eigenvalues of $M_{n}$ in the complex plane in the following sense. For any rectangle $K \subset \mathbb{C}$

$$
\nu\left(K ; M_{n}\right)=\int_{K} d \nu_{M_{n}}
$$

gives the proportion of the eigenvalues of $M_{n}$ that are in $K$. The eigenvalues are counted according to their multiplicity. 
The measure $d \nu_{M_{n}}$ can be obtained from the characteristic polynomial of $M_{n}$ as follows. Let

$$
\begin{aligned}
p\left(z ; M_{n}\right) & =\frac{1}{n} \log \left|\operatorname{det}\left(M_{n}-z I_{n}\right)\right| \\
& =\int_{\mathbb{C}} \log |z-\zeta| d \nu_{M_{n}}(\zeta)
\end{aligned}
$$

In view of $(4.2), p\left(z ; M_{n}\right)$ is the potential of the eigenvalue distribution of $M_{n}$. Obviously, $p\left(z ; M_{n}\right)$ is locally integrable in $z$. Then for any sufficiently smooth function $f(z)$ with compact support

$$
\begin{aligned}
\int_{\mathbb{C}} \log |z-\zeta| \Delta f(z) d^{2} z & =\lim _{\varepsilon \downarrow 0} \int_{|z-\zeta| \geq \varepsilon} \log |z-\zeta| \Delta f(z) d^{2} z \\
& =2 \pi f(\zeta),
\end{aligned}
$$

by Green's formula. Hence

$$
\frac{1}{2 \pi} \int_{\mathbb{C}} p\left(z ; M_{n}\right) \Delta f(z) d^{2} z=\int_{\mathbb{C}} f(z) d \nu_{M_{n}}(z) .
$$

Here $\Delta$ is the two-dimensional Laplacian and $d^{2} z$ is the element of area in the complex plane. Both $p\left(z ; M_{n}\right)$ and $d \nu_{M_{n}}$ define distributions in the sense of the theory of distributions and Eq. (4.3) can be also read as the equality $d \nu_{M_{n}}(z)=\frac{1}{2 \pi} \Delta p\left(z ; M_{n}\right)$ where now $\Delta$ is the distributional Laplacian. More generally, it is proved in potential theory that, under appropriate conditions on $d \nu, d \nu(z)=\frac{1}{2 \pi} \Delta p(z)$, where $p(z)=\int \log |z-\zeta| d \nu_{M_{n}}(\zeta)$ is the potential of $d \nu$. This Poisson's equation relates measures and their potentials.

In this section we shall calculate the limit of $d \nu_{J_{n}}$ for matrices (2.1), obtaining the potential of the limiting measure in terms of the integrated density of states $N(\lambda)$ (see $(2.21)$ ). Our calculation makes use of relation (2.10) according to which the asymmetric matrix $J_{n}$ is, modulo a similarity transformation, a rank 2 perturbation of the symmetric matrix $H_{n}$ (see (2.11)). The low rank of the perturbation allows to obtain explicit formulas describing the change in location of the eigenvalues. In our case,

$$
\operatorname{det}\left(J_{n}-z I_{n}\right)=\operatorname{det}\left(H_{n}+V_{n}-z I_{n}\right)=d\left(z ; H_{n}, V_{n}\right) \operatorname{det}\left(H_{n}-z I_{n}\right),
$$

were

$$
\begin{aligned}
d\left(z ; H_{n}, V_{n}\right) & =\operatorname{det}\left[I_{n}+V_{n}\left(H_{n}-z I_{n}\right)^{-1}\right] \\
& =\left(1+a_{n} G_{n 1}\right)\left(1+b_{n} G_{1 n}\right)-a_{n} b_{n} G_{11} G_{n n}
\end{aligned}
$$

with $a_{n}$ and $b_{n}$ being the top-right and left-bottom corner entries of $V_{n}$ and $G_{l m}$ standing for the $(l, m)$ entry of $\left(H_{n}-z I_{n}\right)^{-1}$.

One can easily obtain (4.6) from (4.5) with the help of a little trick. Write $V_{n}$ in the form $V_{n}=A^{T} B$, where $A$ and $B$ are the following $2 \times n$ matrices:

$$
A=\left(\begin{array}{ccccc}
a_{n} & 0 & \ldots & 0 & 0 \\
0 & 0 & \ldots & 0 & 1
\end{array}\right) \quad B=\left(\begin{array}{ccccc}
0 & 0 & \ldots & 0 & 1 \\
b_{n} & 0 & \ldots & 0 & 0
\end{array}\right) .
$$

Then the $n \times n$ determinant in (4.5) reduces to a $2 \times 2$ determinant, as $\operatorname{det}\left[I_{n}+A^{T} B\left(H_{n}-\right.\right.$ $\left.\left.z I_{n}\right)^{-1}\right]=\operatorname{det}\left[I_{2}+B\left(H_{n}-z I_{n}\right)^{-1} A^{T}\right]$ and the latter, being expanded, gives (4.6). 
Eqs. (4.4) - (4.6) yield the following relationship between the potentials of $d \nu_{J_{n}}$ and $d \nu_{H_{n}}$ :

$$
\begin{aligned}
p\left(z ; J_{n}\right) & =p\left(z ; H_{n}+V_{n}\right) \\
& =p\left(z ; H_{n}\right)+\frac{1}{n} \log \left|d\left(z ; H_{n}, V_{n}\right)\right| .
\end{aligned}
$$

The measures $d \nu_{H_{n}}$ are all supported on the real axis where they converge to the measure $d N(\lambda)$ when $n \rightarrow \infty$. This implies the convergence of their potentials to $\Phi(z)=\int_{\mathbb{R}} \log |z-\lambda| d N(\lambda)$ for all non-real $z$, see Theorem A.2 in Appendix. $\Phi(z)$ is the potential of the limiting distribution of eigenvalues for $H_{n}$. Thus the main part of our calculation of $p\left(z ; H_{n}\right)$ in the limit $n \rightarrow \infty$ is evaluating the contribution of $\frac{1}{n} \log \left|d\left(z ; H_{n}, V_{n}\right)\right|$ to $p\left(z ; J_{n}\right)$. We can do this for all nonreal $z$ lying off the curve $\mathcal{L}$ (see (2.19) and (2.24)). The corresponding result is central to our considerations and we state it as Theorem 4.1 below. Note that, by the Thouless formula (2.22), $\bar{\gamma}(z)=|g|$ is equivalent to $\Phi(z)=\max \left(\mathrm{E} \xi_{0}, \mathrm{E} \eta_{0}\right)$ and thus (cf. $(2.20)$ )

$$
D_{1}=\left\{z: \Phi(z)>\max \left(\mathrm{E} \xi_{0}, \mathrm{E} \eta_{0}\right)\right\} \quad \text { and } \quad D_{2}=\left\{z: \Phi(z)<\max \left(\mathrm{E} \xi_{0}, \mathrm{E} \eta_{0}\right)\right\} .
$$

Theorem 4.1 Assume (2.4)-(2.5). Then for P-almost all $\left\{\xi_{j}, \eta_{j}, q_{j}\right\}_{j=0}^{\infty}$,

$$
\begin{aligned}
\lim _{n \rightarrow \infty} p\left(z ; J_{n}\right) & =\Phi(z) & & \forall z \in D_{1} \backslash \mathbb{R} ; \\
\lim _{n \rightarrow \infty} p\left(z ; J_{n}\right) & =\max \left(\mathrm{E} \xi_{0}, \mathrm{E} \eta_{0}\right) & & \forall z \in D_{2} \backslash \mathbb{R} .
\end{aligned}
$$

The convergence in (4.9) - (4.10) is uniform in $z$ on every compact set in $D_{1} \backslash \mathbb{R}$ and $D_{2} \backslash \mathbb{R}$ respectively.

Proof. In view of Eq. (4.7) and Theorem A.2 in Appendix, we only have to prove that, with probability one, for any compact sets $K_{1} \subset D_{1} \backslash \mathbb{R}$ and $K_{2} \subset D_{2} \backslash \mathbb{R}$

$$
\begin{array}{lll}
\lim _{n \rightarrow \infty} \frac{1}{n} \log \left|d\left(z ; H_{n}, V_{n}\right)\right|=0 & & \text { (uniformly in } \left.z \in K_{1} \subset D_{1} \backslash \mathbb{R}\right), \\
\lim _{n \rightarrow \infty} \frac{1}{n} \log \left|d\left(z ; H_{n}, V_{n}\right)\right|=\max \left(\mathrm{E} \xi_{0}, \mathrm{E} \eta_{0}\right)-\Phi(z) & & \text { (uniformly in } \left.z \in K_{2} \subset D_{2} \backslash \mathbb{R}\right) .
\end{array}
$$

Let us write $d\left(z ; H_{n}, V_{n}\right)$ in the form

$$
d\left(z ; H_{n}, V_{n}\right)=a_{n} G_{n 1}+b_{n} G_{1 n}+a_{n} b_{n} G_{n 1} G_{1 n}+\left(1-a_{n} b_{n} G_{11} G_{n n}\right)
$$

and estimate the four terms on the right-hand side (r.h.s.) of (4.13). Recall that $a_{n}$ and $b_{n}$ are the corner entries of $V_{n}$ and the $G$ 's are the corner entries of $\left(H_{n}-z I_{n}\right)^{-1}$. In particular,

$$
G_{1 n}=G_{n 1}=\frac{\prod_{j=1}^{n-1} c_{j}}{\operatorname{det}\left(H_{n}-z I_{n}\right)}=\frac{e^{\frac{1}{2} \sum_{j=1}^{n-1}\left(\xi_{j}+\eta_{j}\right)}}{\operatorname{det}\left(H_{n}-z I_{n}\right)} .
$$

Under assumptions (2.4) - (2.5), on a set of full probability measure,

$$
a_{n}=-e^{n\left[\frac{1}{2} \mathrm{E}\left(\xi_{0}-\eta_{0}\right)+o(1)\right]}, \quad b_{n}=-e^{-n\left[\frac{1}{2} \mathrm{E}\left(\xi_{0}-\eta_{0}\right)+o(1)\right]},
$$


when $n \rightarrow \infty$ and

$$
e^{\frac{1}{2} \sum_{j=1}^{n-1}\left(\xi_{j}+\eta_{j}\right)}=e^{n\left[\frac{1}{2} \mathrm{E}\left(\xi_{0}+\eta_{0}\right)+o(1)\right]} .
$$

On the other hand,

$$
\left|\operatorname{det}\left(H_{n}-z I_{n}\right)\right|=e^{n p\left(z ; H_{n}\right)}=e^{n\left[\Phi(z)-r\left(z ; H_{n}\right)\right]},
$$

where $r\left(z ; H_{n}\right)=p\left(z ; H_{n}\right)-\Phi(z)$. According to Theorem A.2 in Appendix for P-almost all $\left\{\xi_{j}, \eta_{j}, q_{j}\right\}_{j=0}^{\infty}$

$$
\lim _{n \rightarrow \infty} r\left(z ; H_{n}\right)=0 \quad \text { (uniformly in } z \text { on every compact set } K \subset \mathbb{C} \backslash \mathbb{R} \text { ). }
$$

Therefore, with probability one,

$$
\left|a_{n} G_{n 1}\right|=e^{n\left[\mathrm{E} \xi_{0}-\Phi(z)+o_{z}(1)\right]} \quad \text { and }\left|b_{n} G_{1 n}\right|=e^{n\left[\mathrm{E} \eta_{0}-\Phi(z)+o_{z}(1)\right]},
$$

where the $o_{z}(1)$ terms vanish when $n \rightarrow \infty$ uniformly in $z$ on every compact set $K \subset \mathbb{C} \backslash \mathbb{R}$.

To estimate the third term in the r.h.s. of (4.13), recall the Thouless formula (2.22). As the Lyapunov exponent $\bar{\gamma}(z)$ is non-negative everywhere in the complex plane and $\Phi(z)$, for every fixed $\operatorname{Re} z$, is an increasing function of $|\operatorname{Im} z|$, we have that

$$
\Phi(z)>\frac{1}{2} \mathrm{E}\left(\xi_{0}+\eta_{0}\right) \quad \forall z \in \mathbb{C} \backslash \mathbb{R} .
$$

Moreover, since $\Phi(z)$ is continuous in $z$ off the real axis,

$$
M(K):=\min _{z \in K}\left[\Phi(z)-\frac{1}{2} \mathrm{E}\left(\xi_{0}+\eta_{0}\right)\right]>0
$$

for any compact set $K \subset \mathbb{C} \backslash \mathbb{R}$. From this and (4.16),

$$
\begin{aligned}
\left|a_{n} b_{n} G_{1 n} G_{n 1}\right| & =e^{n\left[E\left(\xi_{0}+\eta_{0}\right)-2 \Phi(z)+o_{z}(1)\right]} \\
& \leq e^{-n\left[2 M(K)+o_{z}(1)\right]} \quad \forall z \in K .
\end{aligned}
$$

In other words, the third term on the r.h.s. in (4.13) vanishes exponentially fast (and uniformly in $z$ on every compact set in $\mathbb{C} \backslash \mathbb{R})$ in the limit $n \rightarrow \infty$.

The fourth term in (4.13) cannot grow exponentially fast with $n$. Nor it can vanish exponentially fast. Estimating it from above is simple. Since $\left|G_{j j}\right| \leq \frac{1}{|\operatorname{Im} z|}, j=1, n$, and $a_{n} b_{n}=e^{o(n)}$, we have, for $P$-almost all $\left\{\xi_{j}, \eta_{j}, q_{j}\right\}_{j=0}^{\infty}$, that

$$
\left|1-a_{n} b_{n} G_{11} G_{n n}\right| \leq 1+\frac{e^{o(n)}}{|\operatorname{Im} z|^{2}} \quad \forall z \in \mathbb{C} \backslash \mathbb{R}
$$

with the $o(n)$ term being independent of $z$.

Estimating $\left|1-a_{n} b_{n} G_{11} G_{n n}\right|$ from below is less trivial. We do this with the help of the two Propositions stated below. The first one is elementary and the second one is a standard result from spectral theory of random operators [5].

Proposition 4.2 Let $C_{\alpha}=\{z \in \mathbb{C}: \quad \alpha \leq \arg z \leq \alpha+\pi\}$, where $0 \leq \alpha \leq \pi$. Then

$$
\min _{z \in C_{\alpha}}|1-z|=\sin \alpha
$$


Proposition 4.3 Under assumptions (2.4) - (2.5), there exists a set $\Omega_{0} \subset \Omega$ of full probability measure such that for every $\omega=\left\{q_{k}, \xi_{k}, \eta_{k}\right\}_{k=0}^{\infty} \in \Omega_{0}$

$$
\lim _{n \rightarrow \infty}\left[\left(H_{n}-z I_{n}\right)^{-1}\right]_{11}=\int_{-\infty}^{+\infty} \frac{d \sigma(\lambda ; \omega)}{\lambda-z} \quad \forall z \in \mathbb{C} \backslash \mathbb{R}
$$

where $\sigma(\lambda ; \omega)$, for every $\omega \in \Omega_{0}$, is a measure on $\mathbb{R}$ with unit total mass. The convergence in (4.21) is uniform on every compact set in $\mathbb{C} \backslash \mathbb{R}$.

Remark. For almost all realizations $\omega$, the semi-infinite matrix

$$
\left(\begin{array}{cccc}
q_{1} & -c_{1} & & \\
-c_{1} & q_{2} & -c_{2} & \\
& \ddots & \ddots & \ddots
\end{array}\right)
$$

where $c_{k}=e^{\frac{1}{2}\left(\xi_{k}+\eta_{k}\right)}$, specifies a selfadjoint operator $H_{+}(\omega)$ on $l^{2}\left(\mathbb{Z}_{+}\right)$. Proposition 4.3 is a consequence of the selfadjointness of $H_{+}(\omega)$, and the measure $\sigma(\lambda ; \omega)$ is simply the $(1,1)$ entry of the resolution of identity for $H_{+}(\omega)$.

Let us set

$$
\alpha\left(z ; H_{n}\right)= \begin{cases}\arg \left[\left(H_{n}-z I_{n}\right)^{-1}\right]_{11}, & \text { if } \operatorname{Im} z>0 \\ \arg \left[\left(z I_{n}-H_{n}\right)^{-1}\right]_{11}, & \text { if } \operatorname{Im} z<0 .\end{cases}
$$

$G_{11}=\left[\left(H_{n}-z I_{n}\right)^{-1}\right]_{11}$, as a function of $z$, maps the upper (lower) half of the complex plane into itself, and so does $G_{n n}$. Therefore,

$$
0<\alpha\left(z ; H_{n}\right)<\pi \quad \forall z \in \mathbb{C} \backslash \mathbb{R}
$$

and

$$
\alpha\left(z ; H_{n}\right) \leq \arg \left(a_{n} b_{n} G_{11} G_{n n}\right) \leq \alpha\left(z ; H_{n}\right)+\pi \quad \forall z \in \mathbb{C} \backslash \mathbb{R} .
$$

Then by Proposition 4.2

$$
\left|1-a_{n} b_{n} G_{11} G_{n n}\right| \geq \sin \alpha\left(z ; H_{n}\right) \quad \forall z \in \mathbb{C} \backslash \mathbb{R} .
$$

Obviously, $\sin \alpha\left(z ; H_{n}\right)=\left|\operatorname{Im} G_{11}\right| /\left|G_{11}\right|$ and by Proposition 4.3 for every $\omega \in \Omega_{0}$ and every compact set $K \subset \mathbb{C} \backslash \mathbb{R}$

$$
\left.\lim _{n \rightarrow \infty} \sin \alpha\left(z ; H_{n}\right)=\frac{\int_{-\infty}^{+\infty} \frac{|\operatorname{Im} z| d \sigma(\lambda ; \omega)}{|\lambda-z|^{2}}}{\left|\int_{-\infty}^{+\infty} \frac{d \sigma(\lambda ; \omega)}{\lambda-z}\right|} \quad \text { (uniformly in } z \in K\right) .
$$

The r.h.s. in (4.22) is positive and continuous in $z \in K$. Therefore it is bounded away from zero uniformly in $z \in K$. Hence, for every $\omega \in \Omega_{0}$ (set of full probability measure) and for every compact set $K \subset \mathbb{C} \backslash \mathbb{R}$

$$
\left.\liminf _{n \rightarrow \infty}\left|1-a_{n} b_{n} G_{11} G_{n n}\right| \geq C(K ; \omega)>0 \quad \text { (uniformly in } z \in K\right),
$$


where the constant $C(K ; \omega)$ depends only on $K$ and $\omega$.

Now we are in a position to prove (4.11) and (4.12). Let $z \in K \subset D_{1} \backslash \mathbb{R}$. Then

$$
\Phi(z)>E \xi_{0} \quad \text { and } \quad \Phi(z)>E \eta_{0} \quad \forall z \in K .
$$

Therefore, by (4.16), the first three terms on the r.h.s. in (4.13) vanish exponentially fast (and uniformly in $z \in K$ ) in the limit $n \rightarrow \infty$. But then, with probability one,

$$
\left.\limsup _{n \rightarrow \infty} \frac{1}{n} \log \left|d\left(z ; H_{n}, V_{n}\right)\right| \leq 0 \quad \text { (uniformly in } z \in K\right),
$$

in view of $(4.20)$, and

$$
\left.\liminf _{n \rightarrow \infty} \frac{1}{n} \log \left|d\left(z ; H_{n}, V_{n}\right)\right| \geq 0 \quad \text { (uniformly in } z \in K\right),
$$

in view of (4.23). This proves (4.11).

Let $K$ be any compact set in $D_{2} \backslash \mathbb{R}$. Then, by the definition of $D_{2}$ and (4.17),

$$
\min \left(\mathrm{E} \xi_{0}, \mathrm{E} \eta_{0}\right)<\Phi(z)<\max \left(\mathrm{E} \xi_{0}, \mathrm{E} \eta_{0}\right)
$$

At this point we may assume, without loss of generality, that $\mathrm{E} \xi_{0} \neq \mathrm{E} \eta_{0}$. For, if $\mathrm{E} \xi_{0}=\mathrm{E} \eta_{0}$ then $D_{2} \backslash \mathbb{R}$ is empty. This follows from (4.17).

Now, if $\mathrm{E} \xi_{0}>\mathrm{E} \eta_{0}$ then $\mathrm{E} \eta_{0}<\Phi(z)<\mathrm{E} \xi_{0}$ for all $z \in K$ and, by (4.16), (4.18) - (4.20), the first term on the r.h.s. in (4.13) dominates the other terms. In this case, with probability one,

$$
\left.\lim _{n \rightarrow \infty} \frac{1}{n} \log \left|d\left(z ; H_{n}, V_{n}\right)\right|=\mathrm{E} \xi_{0}-\Phi(z) \quad \text { (uniformly in } z \in K\right) .
$$

Similarly, if $\mathbf{E} \xi_{0}<\mathrm{E} \eta_{0}$, then it is the second term that dominates and, with probability one,

$$
\left.\lim _{n \rightarrow \infty} \frac{1}{n} \log \left|d\left(z ; H_{n}, V_{n}\right)\right|=\mathrm{E} \eta_{0}-\Phi(z) \quad \text { (uniformly in } z \in K\right) .
$$

Theorem 4.1 is proved.

We shall now deduce from Theorem 4.1 the weak convergence of the eigenvalue distributions $d \nu_{J_{n}}$ to a limiting measure in the limit $n \rightarrow \infty$. In doing this we shall follow Widom [25, 24] who proved that the almost everywhere convergence of the potentials $p\left(z ; M_{n}\right)$ (4.2) of atomic measures $d \nu_{M_{n}}$ implies the weak convergence of the measures themselves provided they are supported inside a bounded domain in the complex plane. Under assumptions (2.4) - (2.5), the spectra of $J_{n}(2.1)$ are not necessarily confined to a bounded domain. To extend Widom's argument to our case we estimate the contribution of the tails of $d \nu_{J_{n}}$ to the corresponding potentials.

Define

$$
p(z)=\max \left[\Phi(z), \mathrm{E} \xi_{0}, \mathrm{E} \eta_{0}\right] .
$$

This function coincides with the r.h.s. of (4.9) - (4.10) and is continuous everywhere in the complex plane except may be the set $\Sigma$, see $(2.26)$. 
$\Phi(z)$ is a subharmonic function [6] and so is $p(z)$. For, the maximum of two subharmonic functions is subharmonic too. Therefore, $\Delta p(z)$ is non-negative in the sense of distribution theory and $\frac{1}{2 \pi} \Delta p(z)$ defines a measure in $\mathbb{C}$ which we denote by $d \nu$,

$$
d \nu(z)=\frac{1}{2 \pi} \Delta p(z)
$$

First, we prove that in the limit $n \rightarrow \infty$ the potentials $p\left(z ; J_{n}\right)$ converge to $p(z)$, for $P$-almost all $\left\{\xi_{k}, \eta_{k}, q_{k}\right\}_{k=0}^{\infty}$, in the sense of distribution theory.

In the Lemma below $C_{0}(\mathbb{C})$ is the space of continuous on $\mathbb{C}$ functions with compact support, and $C_{0}^{\infty}(\mathbb{C})$ is the subspace of $C_{0}(\mathbb{C})$ of those functions which are infinitely differentiable in $\operatorname{Re} z$ and $\operatorname{Im} z$.

Lemma 4.4 Assume (2.4) - (2.5). Then on a set of full probability measure, for every $f \in$ $C_{0}(\mathbb{C})$, and in particular for every $f \in C_{0}^{\infty}(\mathbb{C})$,

$$
\lim _{n \rightarrow \infty} \int_{\mathbb{C}} f(z) p\left(z ; J_{n}\right) d^{2} z=\int_{\mathbb{C}} f(z) p(z) d^{2} z
$$

Proof. Since $p(z)$ is subharmonic, $p(z) \in L_{\text {loc }}^{1}(\mathbb{C})$, so the integral on the r.h.s. of (4.26) makes sense. Let $L_{\delta}=\{z \in \mathbb{C}: \operatorname{dist}(z, \mathbb{R} \cup \mathcal{L}) \leq \delta\}$. By Theorem 4.1, on a set of full probability measure $\Omega_{0}$,

$$
\lim _{n \rightarrow \infty} p\left(z ; J_{n}\right)=p(z) \quad \text { uniformly in } z \text { on compact subsets of } \mathbb{C} \backslash(\mathbb{R} \cup \mathcal{L}) .
$$

It follows from this that on the same set $\Omega_{0}$

$$
\lim _{n \rightarrow \infty} \int_{\mathbb{C} \backslash L_{\delta}} f(z) p\left(z ; J_{n}\right) d^{2} z=\lim _{n \rightarrow \infty} \int_{\mathbb{C} \backslash L_{\delta}} f(z) p(z) d^{2} z
$$

for every continuous $f(z)$ with compact support and for every $\delta>0$.

Since $f(z)$ has compact support, $f(z) p(z) \in L^{1}(\mathbb{C})$. Therefore,

$$
\forall \varepsilon>0 \quad \exists \delta>0: \quad \int_{L_{\delta}}|f(z) p(z)| d^{2} z<\varepsilon
$$

and to complete the proof we only need to show that the same is true for $f(z) p\left(z ; J_{n}\right)$, uniformly in $n$. More precisely, it will suffice to prove the following statement. On a set of full probability measure, for every continuous function $f$ with compact support

$$
\forall \varepsilon>0 \quad \exists \delta>0: \quad \limsup _{n \rightarrow \infty} \int_{L_{\delta}}\left|f(z) p\left(z ; J_{n}\right)\right| d^{2} z<\varepsilon .
$$

Obviously, (4.27) together with (4.28) - (4.29) imply (4.26).

To prove $(4.29)$, we break up $p\left(z ; J_{n}\right)$ into two parts:

$$
\begin{aligned}
p\left(z ; J_{n}\right) & =\frac{1}{n} \sum_{j=1}^{n} \log \left|z_{j}-z\right| \\
& =\frac{1}{n} \sum_{\left|z_{j}\right| \leq R} \log \left|z_{j}-z\right|+\frac{1}{n} \sum_{\left|z_{j}\right|>R} \log \left|z_{j}-z\right|,
\end{aligned}
$$


where $z_{1}, \ldots, z_{n}$ are the eigenvalues of $J_{n}$ and the summation in the two sums in (4.31) is over all eigenvalues of $J_{n}$ satisfying the inequalities $\left|z_{j}\right| \leq R$ and $\left|z_{j}\right|>R$ respectively. The first term in (4.31) is bounded from above but is unbounded from below due to the log-singularities at $z_{j}$. On the contrary, the second term is bounded from below, provided $z$ is separated from the boundary of the disk $|z| \leq R$, but may be unbounded from above due to large values of $\left|z_{j}-z\right|$. We shall treat these two terms separately.

The required estimate on the integral in (4.29) involving the first term in the break-up of $p\left(z ; J_{n}\right)$ (4.31) can be obtained using the property of local integrability of $\log |z|$. Recall that a family of functions $\left\{h_{\alpha}(z)\right\}_{\alpha \in \mathcal{A}}$ is called uniformly integrable in $z$ on a bounded set $D \subset \mathbb{C}$ if for every $\varepsilon>0$ there exists a $\delta>0$ such that for every compact set $S \subset D$ of area less than $\delta$

$$
\int_{S}\left|h_{\alpha}(z)\right| d^{2} z<\varepsilon \quad \forall \alpha \in \mathcal{A}
$$

It is a corollary of the local integrability of $\log |z|$ that for every compact set $K \subset \mathbb{C}$ the family of functions $\{\log |\zeta-z|\}_{\zeta \in K}$ is uniformly integrable in $z$ on bounded subsets of $\mathbb{C}$. From this one immediately obtains

Proposition 4.5 Let $\chi_{R}(|\zeta|)$ be the characteristic function of the disk $|\zeta| \leq R$. For every compact set $K \subset \mathbb{C}$ and for every $R>0$ the family of functions

$$
\left\{\frac{1}{n} \sum_{j=1}^{n} \chi_{R}\left(\left|\zeta_{j}\right|\right) \log \left|\zeta_{j}-z\right|\right\}_{n \geq 1, \zeta_{1}, \ldots, \zeta_{n} \in K}
$$

is uniformly integrable in $z$ on bounded subsets of $\mathbb{C}$.

It is now apparent that for every continuous $f$ with compact support and for every $R>0$

$$
\forall \varepsilon>0 \quad \exists \delta>0: \quad \limsup _{n \rightarrow \infty} \int_{L_{\delta}}\left|f(z) \frac{1}{n} \sum_{\left|z_{j}\right| \leq R} \log \right| z_{j}-z|| d^{2} z<\varepsilon .
$$

To obtain an appropriate upper bound on the second term in (4.31), note the following. If $\zeta$ is such that $\operatorname{dist}\left(\zeta, \operatorname{spec} J_{n}\right) \geq 1$ then $\log |z-\zeta| \geq 0$ for every $z \in \operatorname{spec} J_{n}$ and

$$
\begin{aligned}
0 \leq \sum_{\left|z_{j}\right|>R} \log \left|z_{j}-\zeta\right| & \leq \log \left|\operatorname{det}\left(J_{n}-\zeta I_{n}\right)\right| \\
& \leq \sum_{j=1}^{n} \log \left(e^{\xi_{j-1}}+e^{\eta_{j}}+\left|q_{j}\right|+|\zeta|\right) .
\end{aligned}
$$

The latter inequality is due to the fact that for every matrix $A=\left\|A_{j k}\right\|_{j, k=1}^{n},|\operatorname{det} A| \leq$ $\prod_{j=1}^{n} \sum_{k=1}^{n}\left|A_{j k}\right|$.

By Theorem 2.1, all non-real eigenvalues of $J_{n}$ are in the vicinity of $\mathcal{L}$ for all sufficiently large $n$. $\mathcal{L}$ is a smooth curve and any vertical line in the upper half of the complex plane intersects $\mathcal{L}$ 
only once there. Therefore the probability is one that, moving up along the imaginary axis say, we can find a $\zeta_{0}=i y \operatorname{such}$ that $\operatorname{dist}\left(\zeta_{0}, \operatorname{spec} J_{n}\right) \geq 1$ for all $n \geq n_{0}$.

Note that

$$
\left|\sum_{\left|z_{j}\right|>R} \log \right| z_{j}-z\left|-\sum_{\left|z_{j}\right|>R} \log \right| z_{j}-\zeta_{0}|| \leq \sum_{\left|z_{j}\right|>R}|\log | 1+\frac{\zeta_{0}-z}{z_{j}-\zeta_{0}}||
$$

Choose now $r_{0}$ so that $r_{0}>1$ and the disk $|z| \leq r_{0}$ contains both $\zeta_{0}$ and the support $K$ of $f$. Set $R=4 r_{0}$. Then for all $|z| \leq r_{0}$ and for all $z_{j}$ in the exterior of $|z| \leq R=4 r_{0}$ we have $\left|\zeta_{0}-z\right| \leq 2 r_{0},\left|z_{j}-\zeta_{0}\right| \geq 3 r_{0}$ and $\left|z_{j}-z\right| \geq 3 r_{0}$ and hence

$$
\begin{aligned}
\left|\frac{1}{n} \sum_{\left|z_{j}\right|>R} \log \right| z_{j}-z|| & \leq \log 3+\left|\frac{1}{n} \sum_{\left|z_{j}\right|>R} \log \right| z_{j}-\zeta_{0}|| & \forall|z| \leq r_{0} \\
& \leq \log 3+\frac{1}{n} \sum_{j=1}^{n} \log \left(e^{\xi_{j-1}}+e^{\eta_{j}}+\left|q_{j}\right|+r_{0}\right) & {[\text { by }(4.33-(4.34))] . }
\end{aligned}
$$

By the ergodic theorem, under assumptions (2.4) - (2.5), on a set $\Omega_{1}$ of full probability measure,

$$
\lim _{n \rightarrow \infty} \frac{1}{n} \sum_{j=1}^{n} \log \left(e^{\xi_{j-1}}+e^{\eta_{j}}+\left|q_{j}\right|+r_{0}\right)=\mathrm{E} \log \left(e^{\xi_{0}}+e^{\eta_{1}}+\left|q_{1}\right|+r_{0}\right)<+\infty
$$

Therefore, on $\Omega_{1}$,

$$
\limsup _{n \rightarrow \infty}\left\{\frac{1}{n} \sup _{|z| \leq r_{0}}\left|\sum_{\left|z_{j}\right|>R} \log \right| z_{j}-z||\right\} \leq \text { Const }
$$

and, because the disk $|z| \leq r_{0}$ covers the support of $f$,

$$
\forall \varepsilon>0 \quad \exists \delta>0: \quad \limsup _{n \rightarrow \infty} \int_{L_{\delta}}\left|f(z) \frac{1}{n} \sum_{\left|z_{j}\right|>R} \log \right| z_{j}-z|| d^{2} z<\varepsilon .
$$

(4.32) and (4.35) imply (4.29). Lemma 4.4 is proved.

Corollary 4.6 Assume (2.4) - (2.5). Then with probability one,

$$
\lim _{n \rightarrow \infty} d \nu_{J_{n}}=d \nu
$$

in the sense of weak convergence of measures.

Proof. Since the operation $\Delta$ is continuous on distributions, Lemma 4.4 implies that, on a set of full probability measure,

$$
d \nu_{J_{n}}(z)=\frac{1}{2 \pi} \Delta p\left(z ; J_{n}\right) \rightarrow \frac{1}{2 \pi} \Delta p(z)=d \nu(z), \text { as } n \rightarrow \infty,
$$


as distributions. To complete the proof, recall that a sequence of measures converging as distributions must be converging weakly [12].

It is apparent that $\Delta p(z)=0$ everywhere off a line consisting of two parts. One is the equipotential line $\mathcal{L}(2.24)$ that separates the domains $D_{1}$ and $D_{2}$; the other is $\Sigma(2.26)$ which is made up of all points of $\operatorname{Supp} d N$ that do not belong to the interior of the closed contours of $\mathcal{L}$.

$p(z)$ is continuous in the upper and lower parts of the complex plane. It also has continuous derivatives everywhere but on $\mathcal{L}$ and $\Sigma$. Its normal derivative has a jump when $z$ moves from $D_{2}$ to $D_{1}$ in the direction perpendicular to $\mathcal{L}$. It follows from this that the restriction $d \nu^{C}$ of $d \nu$ to $\mathbb{C} \backslash \mathbb{R}$ is supported on $\mathcal{L}$ and has there density $\rho(z)$ with respect to the arc-length measure $d l$ on $\mathcal{L}$. The density equals the jump in the normal derivative of $p(z)$ multiplied by $\frac{1}{2 \pi}$. A straightforward calculation gives

$$
\rho(z)=\frac{1}{2 \pi}\left|\int_{-\infty}^{+\infty} \frac{d N(\lambda)}{\lambda-z}\right|
$$

On the other hand, the restriction $d \nu^{R}$ of $d \nu$ to the real axis is supported on $\Sigma$ and coincides there with $d N$. Therefore, for every bounded continuous function $f(z)$

$$
\begin{aligned}
\int_{\mathbb{C}} f(z) d \nu(z) & =\int_{\mathbb{C}} f(z) d \nu^{R}(z)+\int_{\mathbb{C}} f(z) d \nu^{C}(z) \\
& =\int_{\Sigma} f(\lambda) d N(\lambda)+\int_{\mathcal{L}} f(z(l)) \rho(z(l)) d l .
\end{aligned}
$$

Taking into account that the weak convergence of measures $d \nu_{n}$ is equivalent to the convergence of $\int f(z) d \nu_{n}(z)$ on bounded continuous functions we obtain from (4.36) - (4.37) and Corollary 4.6 the statement of Theorem 2.2.

\section{A Appendix}

In our analysis of the non-self-adjoint eigenvalue problem (2.7)-(2.8) we have used a number of results about the finite difference equation

$$
-c_{k-1} \varphi_{k-1}-c_{k} \varphi_{k+1}+q_{k} \varphi_{k}=z \varphi_{k}
$$

with random coefficients $c_{k}$ and $q_{k}$. For the sake of completeness, we reproduce here the corresponding formal statements. With the exception of Theorem A.4 these results are well known in the theory of random selfadjoint operators. Their proofs together with references to the original publications can be found in books $[5,18]$. Some of the results are proved there under slightly less general assumptions than those used in this paper. However only minor adjustments are needed to extend the published proofs to the generality of our assumptions.

Given a sequence of 2-component vectors $\left\{\left(c_{k}, q_{k}\right)\right\}_{j=1}^{\infty}$, consider a sequence of tridiagonal symmetric matrices $H_{n}$ of dimension $n, n=1,2, \ldots$, defined on $\left\{\left(c_{k}, q_{k}\right)\right\}_{j=1}^{\infty}$ in the following way

$$
H_{n}=\left(\begin{array}{cccc}
q_{1} & -c_{1} & & \\
-c_{1} & \ddots & \ddots & \\
& \ddots & \ddots & -c_{n-1} \\
& & -c_{n-1} & q_{n}
\end{array}\right)
$$


Assume that

$\omega \equiv\left\{\left(c_{j}, q_{j}\right)\right\}_{j=1}^{\infty}$ is a stationary ergodic (with respect to the translation $k \rightarrow k+1$ ) sequence of random vectors defined on a common probability space $(\Omega, \mathcal{F}, P)$;

$c_{k}>0$ for all $k$, and $\mathrm{E} \ln \left(1+\left|q_{1}\right|\right)<+\infty, \mathrm{E}\left|\ln c_{1}\right|<+\infty$.

Here as before the symbol $\mathrm{E}$ denotes averaging ever the probability space.

The matrix $H_{n}$ is symmetric and has real eigenvalues. Their empirical cumulative distribution function is defined as

$$
N_{n}(\lambda, \omega)=\frac{1}{n} \#\left\{\text { eigenvalues of } H_{n} \text { in }(-\infty, \lambda)\right\} .
$$

Theorem A.1 Assume (A.2) - (A.3). Then there exists a continuous non-random function $N(\lambda)$ of real variable $\lambda$ such that for almost all sequences $\omega$

$$
\lim _{n \rightarrow \infty} N_{n}(\lambda, \omega)=N(\lambda) .
$$

In other words, on a set of full probability measure, the eigenvalue counting measures $d N_{n}(\lambda, \omega)$ converge weakly, as $n \rightarrow \infty$, to the limiting measure $d N(\lambda)$.

The limit distribution function, $N(\lambda)$, is called the integrated density of states (IDS) of Eq. (A.1).

Let

$$
p\left(z, H_{n}\right)=\int_{-\infty}^{+\infty} \log |\lambda-z| d N_{n}(\lambda, \omega)
$$

and

$$
\Phi(z)=\int_{-\infty}^{+\infty} \log |\lambda-z| d N(\lambda)
$$

Under assumptions (A.2) - (A.3), the integral in (A.5) converges for every non-real $z$, hence $\Phi(z)$ is well defined off the real axis. On the real axis, the equality in (A.5) is understood in the following sense ${ }^{4}$

$$
\Phi(x)=\lim _{\varepsilon \downarrow 0} \int_{-\infty}^{+\infty} \max \left[\log |\lambda-x|,-\varepsilon^{-1}\right] d N(\lambda), \quad x \in \mathbb{R}
$$

with the convention that $\Phi(x)=-\infty$ if the above limit is $-\infty^{5}$. With this convection, $\Phi(z)$ is subharmonic in the complex plane. In particular, $\Phi(z)$ is upper semi-continuous.

Theorem A.2 Assume (A.2) - (A.3). Then the following is true for almost all $\omega$ : For all non-real z

$$
\lim _{n \rightarrow \infty} p\left(z ; H_{n}\right)=\Phi(z) .
$$

The convergence in (A.6) is uniform in z on every compact subset of $\mathbb{C} \backslash \mathbb{R}$.

\footnotetext{
${ }^{4}$ Under assumptions (A.2) - (A.3), the prelimit integral converges for every $\varepsilon>0$

${ }^{5}$ In fact, $\Phi(z)$ is finite everywhere in the complex plane, see (A.9).
} 
Let

$$
\gamma_{n}(z, \omega)=\frac{1}{n} \log \left\|S_{n}(z)\right\|
$$

where $S_{n}(z)$ is as in $(2.15)$.

The Lyapunov exponent is defined as follows:

$$
\bar{\gamma}(z)=\lim _{n \rightarrow \infty} \mathrm{E} \gamma_{n}(z, \omega)
$$

The limit above exists for every $z \in \mathbb{C}$. Any matrix norm can be used in (A.7), as they all are equivalent. Since $\left\|S_{n}\right\|^{2} \geq\left|\operatorname{det} S_{n}\right|$ and $\operatorname{det} S_{n}(z)=c_{0} / c_{n}$, we have that for every $z \in \mathbb{C}$, and in particular for every real $z$,

$$
\bar{\gamma}(z) \geq 0 \text {. }
$$

Theorem A.3 Assume (A.2) - (A.3). Then for almost all $\omega$ the following is true: For every compact set $K \subset \mathbb{C} \backslash \mathbb{R}$

$$
\lim _{n \rightarrow \infty} \gamma_{n}(z, \omega)=\bar{\gamma}(z) \quad \text { uniformly in } z \in K
$$

Remark. In contrast to non-real $z$, on the real axis

$$
\lim _{n \rightarrow \infty} \gamma_{n}(x, \omega)=\gamma(x, \omega)
$$

The limit above exists for almost all pairs $(x, \omega)$ and $\mathrm{E} \gamma(x, \omega)=\bar{\gamma}(z)$. However, when $x$ is fixed, the set $\Omega_{x}$ of those $\omega$ for which the limit in (A.7) exists depends on $x$, and $P\left(\cap_{x \in \Sigma} \Omega_{x}\right)=0$ (see $[14,15,1])$.

For every $\omega, \gamma_{n}(z, \omega)$ is a subharmonic function in the complex plane. $\bar{\gamma}(z)$ is also subharmonic in $\mathbb{C}$. This property of the Lyapunov exponent is very useful, see [6]. We use it here to deduce the following corollary from Theorem A.3.

Theorem A.4 Assume (A.2) - (A.3). Then for almost all $\omega$ the following is true: For every compact set $K \subset \mathbb{C}$, and in particular for every compact set $K \subset \mathbb{R}$,

$$
\limsup _{n \rightarrow \infty}\left\{\sup _{z \in K} \gamma_{n}(z, \omega)\right\} \leq \sup _{z \in K} \bar{\gamma}(z) .
$$

If, in addition, $\bar{\gamma}(z)$ is continuous in $K$ then

$$
\limsup _{n \rightarrow \infty}\left\{\sup _{z \in K}\left[\gamma_{n}(z, \omega)-\bar{\gamma}(z)\right]\right\} \leq 0
$$


Remark. This theorem plays a crucial role in our proof of the fact that the eigenvalues of $J_{n}$ are wiped out, as $n \rightarrow \infty$, from the interior of each contour of the curve $\mathcal{L}$, see part (b) of Theorem 2.1. Actually, our proof of part (a) of Theorem 2.1 also applies to any compact subset of $D_{2} \backslash \mathbb{R}$. However, this proof is based on Theorem A.3 and cannot be applied to the whole interior of $\mathcal{L}$ as it contains intervals of real axis.

Theorem A.4 follows immediately from Theorem A.3 and the following result from the theory of subharmonic functions:

Theorem A.5 [see [13], p. 150] Let $u_{j} \not \equiv-\infty$ be a sequence of subharmonic functions in $\mathbb{C}$ converging in the sense of distribution theory to the subharmonic function $u$. If $K$ is a compact subset of $\mathbb{C}$ and $f$ is continuous on $K$, then

$$
\limsup _{j \rightarrow \infty}\left\{\sup _{K}\left(u_{j}-f\right)\right\} \leq \sup _{K}(u-f)
$$

Theorem A.6 (Thouless formula) For all $z \in \mathbb{C}, \bar{\gamma}(z)=\Phi(z)-\mathrm{E} \log c_{1}$.

It is a corollary of the Thouless formula and the positivity of the Lyapunov exponent $\bar{\gamma}(z)$ that

$$
\Phi(z) \geq \mathrm{E} \log c_{0} \quad \forall z \in \mathbb{C} .
$$

\section{References}

[1] Avron, J. and Simon, B. (1983), Almost Periodic Schrödinger Operators II., Duke Math. Journ. 50, $369-391$.

[2] Bougerol, P. and Lacroix, J. (1995), Products of Random Matrices with Applications to Random Schrödinger Operators. Birkhäuser, Boston.

[3] Brezin, E. and Zee, A. (1998), Non-Hermitian localization: Multiple scattering and bounds. Nucl. Phys. B509[FS], $599-614$.

[4] Brouwer P. W., Silvestrov, P. G., and Beenakker, C. W. J. (1997), Theory of directed localization in one dimension. Phys. Rev. B56, R4333 - R4336.

[5] Carmona, R. and Lacroix, J. (1990), Spectral Theory of Random Schrödinger Operators. Birkhäuser, Boston.

[6] Craig, W. and Simon, B. (1983), Subharmonicity of the Lyapunov Index. Duke Math. Journ. 50 (1983), $551-560$.

[7] Davies, E. B., Spectral properties of non-self-adjoint matrices and operators. To appear in Proc. Royal Soc. A. 
[8] Davies, E. B., Spectral theory of pseudo-ergodic operators. To appear in Commun. Math. Phys.

[9] Hatano N. and Nelson, D. R. (1996), Localization transitions in non-Hermitian quantum mechanics. Phys. Rev. Lett. 77, $570-573$.

[10] Hatano N. and Nelson D. R. (1997), Vortex pinning and non-Hermitian quantum mechanics. Phys. Rev. B56, $8651-8673$.

[11] Hirshman Jr., I. I. (1967), The spectra of certain Toeplitz matrices. Illinois J. Math. 11, $145-159$.

[12] Hörmander, L. (1983), The analysis of linear partial differential equations, Vol. I. Springer, New York.

[13] Hörmander, L. (1994) Notions of Convexity. Birkhäuser, Boston.

[14] Goldsheid, I. Ya. (1975), Asymptotic behaviour of a product of random matrices that depend on a parameter. Dokl. Akad. Nauk. SSSR 224, 1248 - 1251.

[15] Goldsheid, I. Ya. (1980), Asymptotic properties of the product of random matrices depending on a parameter. In Adv. in Prob. 8, pp. 239 - 283, Ya. G. Sinai and R. Dobrushin, eds. Dekker, New York.

[16] Goldsheid I. Ya. and Khoruzhenko, B. A. Distribution of eigenvalues in non-Hermitian Anderson models. Phys. Rev. Lett. 80, 2897 - 2900 (1998).

[17] Naiman P. B. (1964), On the spectral theory of non-symmetric periodic Jacobi matrices (in Russian). Zap. Meh.-Mat. Fak. Har' kov. Gos. Univ. i Har'kov. Mat. Obsc., (4), 30, 138 151.

[18] Pastur, L. A. and Figotin, A. L. (1992), Spectra of Random and Almost-Periodic Operators. Springer, Berlin.

[19] Reichel, L. and Trefethen, L. N. (1992), Eigenvalues and pseudo-eigenvalues of Toeplitz matrices. Lin. Alg. Appl. 162-4, 153 - 185.

[20] Schmidt, P. and Spitzer, F. (1960), The Toeplitz matrices of an arbitrary Laurent polynomial. Math. Scand. 8, 15 - 38.

[21] Trefethen, L. N. (1991) Pseudospectra of matrices. In D. F. Numerical Analysis 1991, pp. 336 - 360, Griffiths and G. A. Watson, eds. Longman Scientific and Technical, Harlow, Essex, UK, 1992.

[22] Trefethen, L. N. Contendini, M., and Embree, M., Spectra, pseudospectra, and localization for random bidiagonal matrices. To appear in Commun. Pure and Appl. Math.

[23] Trefethen, L. N., Trefethen, A. E., Reddy, S. C., and Driscoll, T. A. (1993), Hydrodynamic stability without eigenvalues. Science 261, $578-584$.

[24] Widom, H. (1994), Eigenvalue distribution for nonselfadjoint Toeplitz matrices. In Operator Theory: Advances and Applications Vol. 71, pp. 1 - 8. 
[25] Widom, H. (1990), Eigenvalue distribution of nonselfadjoint Toeplitz matrices and the asymptotics of the Toeplitz determinants in the case of nonvanishing index. In Operator Theory: Advances and Applications Vol. 48, pp. 387 - 421. 